ESAIM: M2AN 51 (2017) 1279-1301

DOI: $10.1051 / \mathrm{m} 2 \mathrm{an} / 2016060$
ESAIM: Mathematical Modelling and Numerical Analysis

www.esaim-m2an.org

\title{
NEW TRANSMISSION CONDITION ACCOUNTING FOR DIFFUSION ANISOTROPY IN THIN LAYERS APPLIED TO DIFFUSION MRI
}

\author{
Fabien Caubet ${ }^{1}$, Houssem Haddar ${ }^{2}$, Jing-Rebecca LI $^{2}$ and Dang Van Nguyen ${ }^{2}$
}

\begin{abstract}
The Bloch-Torrey Partial Differential Equation (PDE) can be used to model the diffusion Magnetic Resonance Imaging (dMRI) signal in biological tissue. In this paper, we derive an Anisotropic Diffusion Transmission Condition (ADTC) for the Bloch-Torrey PDE that accounts for anisotropic diffusion inside thin layers. Such diffusion occurs, for example, in the myelin sheath surrounding the axons of neurons. This ADTC can be interpreted as an asymptotic model of order two with respect to the layer thickness and accounts for water diffusion in the normal direction that is low compared to the tangential direction. We prove uniform stability of the asymptotic model with respect to the layer thickness and a mass conservation property. We also prove the theoretical quadratic accuracy of the ADTC. Finally, numerical tests validate these results and show that our model gives a better approximation of the dMRI signal than a simple transmission condition that assumes isotropic diffusion in the layers.
\end{abstract}

Mathematics Subject Classification. 35C20, 35Q92.

Received March 30, 2015. Revised June 29, 2016. Accepted September 8, 2016.

\section{INTRODUCTION}

Diffusion Magnetic Resonance Imaging (dMRI) gives a measure of the average distance travelled by water molecules in a medium and can give useful information on cellular structure and structural change when the medium is biological tissue. A large number of works have appeared in recent years that show that dMRI measurements can be correlated with various physiological or pathological conditions such as cell swelling, demyelinating disorders or the presence of tumors (see, e.g., $[18,20,21,23,27]$ and references therein). In particular, dMRI can be used to detect and quantify abnormalities in the myelin sheath surrounding the axons of neurons (see $[4,7,12,13])$. The loss of or damage to the myelin sheath can be correlated with many diseases of brain function.

A commonly used mathematical model for water proton magnetization in tissue is the Bloch-Torrey (see [35]) Partial Differential Equation (PDE), where intrinsic diffusion tensors are defined in different cellular geometrical compartments. In this paper, we start with a three-compartment geometrical model: the three geometrical compartments are (1) the axons, (2) myelin sheath surrounding the axons, (3) the extra-cellular space. We want

\footnotetext{
Keywords and phrases. Asymptotic expansion, Bloch-Torrey equation, anisotropic diffusion transmission condition, diffusion magnetic resonance imaging.

1 Institut de Mathématiques de Toulouse, Université de Toulouse, 31062 Toulouse cedex 9, France.

fabien. caubet@math. univ-toulouse.fr

2 INRIA Saclay, Équipe DéFI, CMAP, École Polytechnique, Route de Saclay, 91128 Palaiseau, France
} 


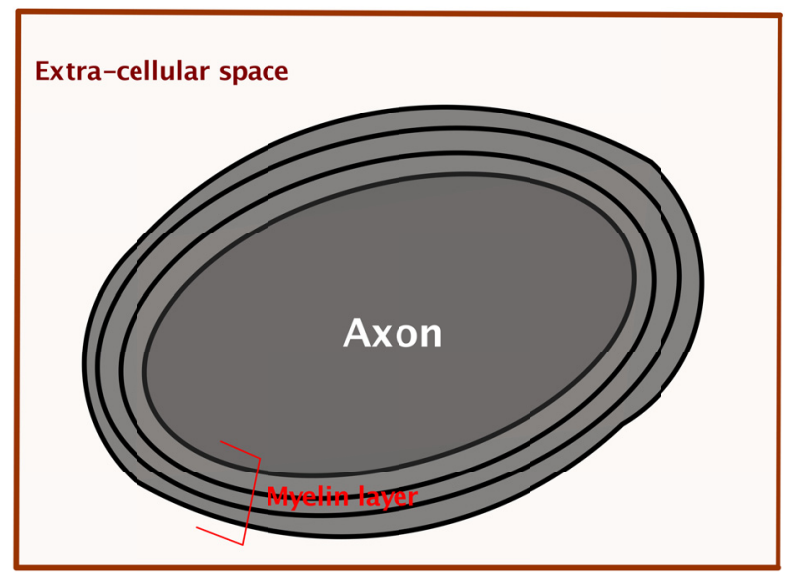

FIGURE 1. Illustration of the myelin sheath, composed of layers of lipids, surrounding the axon.

to approximate this original three-compartment model by a two-compartment geometrical model: the two geometrical compartments are (1) the axons and (2) the extra-cellular space. These two compartments will be linked via a transmission condition.

To obtain asymptotic two-compartment models, we rely on a methodology based on classical scaled asymptotic expansions for thin structures (see $[8,10,30]$ ) and on an appropriate scaling of tangential and normal diffusion inside the myelin layer. This methodology has been extensively used to model thin coatings (see, e.g., $[6,15]$ and references therein), rough boundaries (see, e.g., $[1,19]$ and references therein) and imperfectly conducting obstacles (see, e.g., [16,17] and references therein). We can also mention here the recent works [11,29].

A simple and well-known transmission condition can be used when the diffusion inside the layer is isotropic. However, since the myelin sheath is composed of layers of lipids or proteins [31] (see Fig. 1 for an illustration), it is expected that the diffusion tensor in the myelin sheath will have a normal component that is much smaller than the tangential component (see discussion about diffusion inside the myelin sheath in $[5,22,24]$ ).

To account for low diffusion normal to the layer, we make the following choice for the diffusion inside the layer:

(1) for the tangential direction, we use the same scaling as for diffusion in the axons and in the extra-cellular space;

(2) for the normal direction, we use a scaling proportional to the layer thickness.

This choice leads to asymptotic transmission conditions. The first order approximation (in the layer thickness) leads to a transmission condition that has the same form as the classical transmission condition associated with isotropic layer diffusion. Anisotropy appears in the second order approximation and gives rise to our new Anisotropic Diffusion Transmission Condition (ADTC). This ADTC couples volumetric diffusion equations with surface diffusion equations. We note that the natural expression of the second order transmission condition does not exhibit uniform time stability with respect to the layer thickness, but this well-known phenomenon for higher order asymptotic models can be corrected by the use of a Padé expansion, as in $[8,14]$, and our ADTC is corrected in this way. Thus, in its final form, our ADTC has a mass-conservation property, which is important for dMRI modeling. We also give a proof of error estimates for the obtained second order approximate model.

We implemented a finite elements discretization of the new ADTC in two dimensions and conducted numerical tests that confirm second order accuracy with respect to layer thickness. The ADTC that we propose here simplifies the numerical solution of the dMRI model (i.e. solving the Bloch-Torrey equation at a microscopic 
scale) by removing the need to discretize the myelin sheath, which results in a computational saving that may be significant in three dimensions when simulating arbitrarily oriented white matter fibers.

The paper is organized as follows. We first explain the problem setting and describe the Bloch-Torrey equation in Section 2. Then, in Section 3, we detail the asymptotic method to obtain the new ADTC and prove uniform stability of the new model with respect to the layer thickness and a mass conservation property. Our numerical results are presented in Section 4. Conclusions are in Section 5. We present in an appendix the error analysis for the asymptotic model obtained in Section 3.3.2.

\section{Bloch-TORREy EQUATION TO MODEL THE DIFFUSiOn MRI SIGNAL}

A classic dMRI experiment consists of applying two pulsed gradient magnetic fields with a 180 degree spin reversal between the two pulses in order to encode the displacement of the water molecules between the two pulses (see, e.g., [34]). The complex transverse water proton magnetization $M$ can be modeled by the following Bloch-Torrey PDE (see, e.g., [35]):

$$
\frac{\partial M(\mathbf{x}, t)}{\partial t}+i \mathbf{q} \cdot \mathbf{x} f(t) M(\mathbf{x}, t)-\operatorname{div}(\overline{\boldsymbol{\sigma}}(\mathbf{x}) \nabla M(\mathbf{x}, t))=0,
$$

where $i:=\sqrt{-1}, \overline{\boldsymbol{\sigma}}(\mathbf{x})$ is the intrinsic diffusion tensor, $\mathbf{q}$ contains the amplitude and direction information of the applied diffusion-encoding magnetic field gradient multiplied by the gyro-magnetic ratio of the water proton, and $f$, where $\max _{t} f(t)=1$, is the normalized time profile of the diffusion-encoding magnetic field gradient sequence. The time profile of the classic Pulsed Gradient Spin Echo (PGSE) [34] sequence (simplified to include only the parameters relevant to diffusion, i.e., the imaging gradients are ignored) is the following:

$$
f(t):= \begin{cases}1, & 0<t \leq \delta \\ -1, & \Delta<t \leq \Delta+\delta \\ 0, & \text { elsewhere }\end{cases}
$$

where $0 \leq \delta \leq \Delta$ and where we made $f(t)$ negative in the second pulse to include the effect of the 180 degree spin reversal between the pulses. The time at which the signal is measured is called the echo time $T E>\delta+\Delta$.

The dMRI signal is the total magnetization:

$$
S(\mathbf{q}):=\int M(\mathbf{x}, \delta+\Delta) \mathrm{d} \mathbf{x},
$$

where $M$ is the solution of equation (2.1). The signal is usually plotted against a quantity called the $b$-value, given by

$$
b(\mathbf{q}):=\|\mathbf{q}\|^{2} \delta^{2}\left(\Delta-\frac{\delta}{3}\right),
$$

because for a homogeneous domain, where $\overline{\boldsymbol{\sigma}}(\mathbf{x})=\overline{\boldsymbol{\sigma}}$ is constant, the signal has the analytical expression

$$
S(\mathbf{q})=\exp \left(-\left(\frac{\mathbf{q}^{T} \overline{\boldsymbol{\sigma}} \mathbf{q}}{\|\mathbf{q}\|^{2}}\right) b(\mathbf{q})\right)
$$

where the quantity before the $b$-value is the diffusion coefficient in the direction of $\mathbf{q}$.

\subsection{Geometrical compartments}

A standard geometrical model of the brain white matter (for an early example, see [3]) divides the tissue into three compartments:

(1) $\Omega_{i}^{\eta}$ is the axons (with associated intrinsic diffusion tensor $\overline{\boldsymbol{\sigma}}_{i}$ ); 


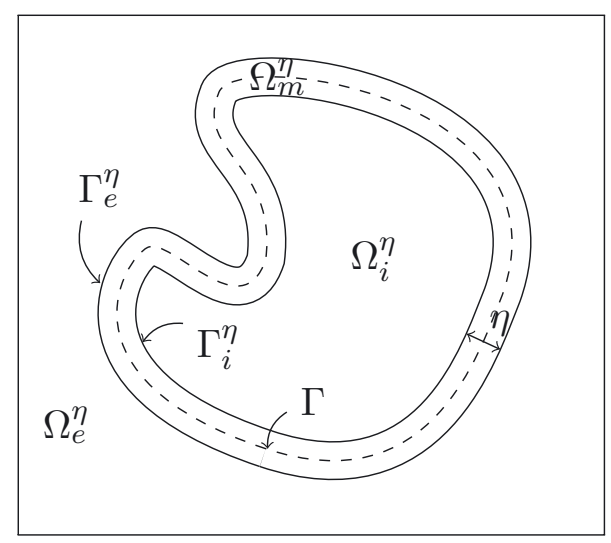

(a)

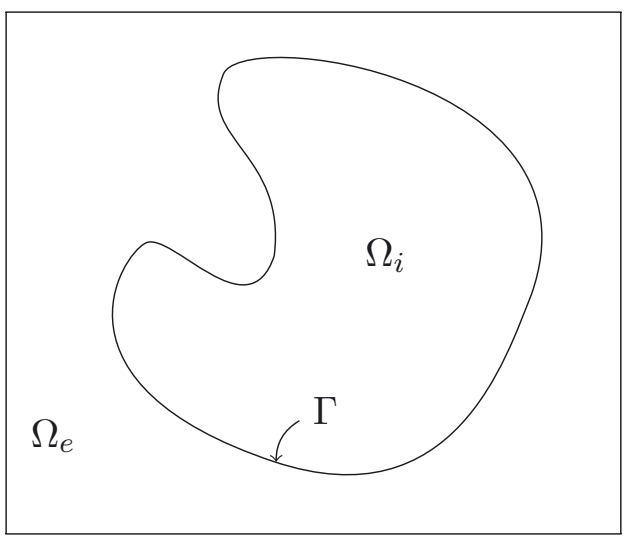

(b)

Figure 2. Notations for the three compartment model (left) and the two compartment model (right).

(2) $\Omega_{e}^{\eta}$ is the extra-cellular space (with associated intrinsic diffusion tensor $\overline{\boldsymbol{\sigma}}_{e}$ );

(3) $\Omega_{m}^{\eta}$ is the myelin sheath (with associated intrinsic diffusion tensor $\overline{\boldsymbol{\sigma}}_{m}$ ).

We denote by $\eta$ the thickness of the layer (which is assumed to be constant) and by $\Gamma$ a fictitious interface inside the myelin layer at equal distance from the two boundaries of the layer. We denote by $\Omega$ the domain formed by union of $\Omega_{\ell}^{\eta}, \ell=e, i, m$. We also introduce some notations as we consider the geometrical compartments when $\eta \rightarrow 0$ : we denote the remaining two compartments by $\Omega_{i}$ and by $\Omega_{e}$ (see Fig. 2). For the ease of notation, we restrict the diffusion tensor, $\overline{\boldsymbol{\sigma}}(\mathbf{x})$, for the tissue to be piece-wise constant:

$$
\overline{\boldsymbol{\sigma}}(\mathbf{x}):= \begin{cases}\overline{\boldsymbol{\sigma}}_{i}(\mathbf{x}), & \mathbf{x} \in \Omega_{i}^{\eta}, \\ \overline{\boldsymbol{\sigma}}_{e}(\mathbf{x}), & \mathbf{x} \in \Omega_{e}^{\eta}, \\ \overline{\boldsymbol{\sigma}}_{m}(\mathbf{x}), & \mathbf{x} \in \Omega_{m}^{\eta} .\end{cases}
$$

For the three compartment model, the natural continuity conditions (of the magnetization and the flux) on the compartment interfaces result is the following Interface Conditions (IC) on the boundaries $\Gamma_{i}^{\eta}$ and $\Gamma_{e}^{\eta}$ of $\Omega_{i}^{\eta}$ and $\Omega_{e}^{\eta}$ :

$$
\text { IC on } \Gamma_{\mathrm{e}}^{\eta} \text { and } \Gamma_{i}^{\eta}: \begin{cases}{[\overline{\boldsymbol{\sigma}} \nabla M \cdot \mathbf{n}]} & =0, \\ {[M]} & =0,\end{cases}
$$

where $\mathbf{n}$ is the normal to $\Gamma_{e}^{\eta}$ or $\Gamma_{i}^{\eta}$. The symbol [.] denotes the jump relative to the direction of $\mathbf{n}$.

Finally, the anisotropy inside the layer is assumed to be such that

$$
\overline{\boldsymbol{\sigma}}_{m}:\left\{\begin{array}{l}
\overline{\boldsymbol{\sigma}}_{m} \mathbf{n}=\sigma_{m}^{n} \mathbf{n}, \\
\overline{\boldsymbol{\sigma}}_{m} \boldsymbol{\tau}=\sigma_{m}^{\tau} \boldsymbol{\tau},
\end{array}\right.
$$

where $\sigma_{m}^{n}$ and $\sigma_{m}^{\tau}$ indicate respectively the transverse diffusion coefficient and tangential diffusion coefficient in the layer and $\tau$ represents the unit tangential vector. The important assumption we make in this paper (see (3.4) below) implies that

$$
\sigma_{m}^{n} \ll \sigma_{m}^{\tau}
$$




\subsection{Classical asymptotic model for isotropic diffusion in layer}

If the diffusion inside the layer is isotropic, i.e. $\sigma_{m}^{n}=\sigma_{m}^{\tau}$, it is well-known that the following asymptotic transmission condition, which we denote the Isotropic Diffusion Transmission Condition (IDTC), can be imposed on the interface $\Gamma$ (see, e.g., [9]):

$$
\text { IDTC on } \Gamma:\left\{\begin{array}{l}
{[\overline{\boldsymbol{\sigma}} \nabla M \cdot \mathbf{n}]=0,} \\
\overline{\boldsymbol{\sigma}} \nabla M \cdot \mathbf{n}=\kappa_{0}[M]
\end{array}\right.
$$

where $\kappa_{0}$ is a given permeability coefficient. We recall that in addition to equation (2.10), the PDE (2.1) is assumed to hold on $\Omega^{i}$ and $\Omega^{e}$. As we shall see, this type of transmission condition corresponds to a first order asymptotic model when the diffusion tensor inside the membrane scales like $\eta$. Our goal is to improve this condition by taking into account the $O(1)$ tangential diffusion.

\section{Formal DERIVATION OF TRANSMISSION CONDITIONS}

The methodology we shall adopt to derive transmission conditions is similar to the one in $[6,15,16]$ and is based on a scaling of the layer with respect to its thickness $\eta$ and an asymptotic expansion of the fields with respect to $\eta$. We shall restrict ourselves in this section to a formal obtention of these conditions (in the sense that no convergence proof will be established). The latter is technical and is usually valid (for linear problems) as long as the obtained model is proved to be uniformly stable with respect to the thickness. This is why we shall only discuss this last point in the present section. For a sketch of the convergence proof, we refer the reader to the appendix. The following formal technical details in space dimension 2 are largely inspired by [2] (see also [16] for space dimension 3).

\subsection{Expression of the differential operators in curvilinear coordinates}

We assume that $\Gamma$ is a regular curve (at least $C^{2}$ ) and is the boundary of a simply connected domain $\Omega_{i}$ (independent from $\eta$ ). Notice that we can treat the case of multiply connected domains by treating separately each connected component. Then, the boundary $\Gamma$ can be parametrized in terms of the curvilinear abscissa $s$ as $s \mapsto \mathbf{x}_{\Gamma}(s), s \in\left[0, L\left[\right.\right.$, with $\left|\mathrm{d} \mathbf{x}_{\Gamma}(s) / \mathrm{d} s\right|=1$, where $L$ is the length of $\Gamma$. We assume that this parametrization defines a clockwise orientation. Let $\mathbf{n}(s)$ be the unitary normal vector at $\mathbf{x}_{\Gamma}(s)$ directed to the exterior of $\Omega_{i}$ and set $\boldsymbol{\tau}(s)=\mathrm{d} \mathbf{x}_{\Gamma}(s) / \mathrm{d} s$ which is a unitary vector tangential to $\Gamma$ at $\mathbf{x}_{\Gamma}(s)$. The curvature $c$ can be defined by

$$
c(s):=\boldsymbol{\tau}(s) \cdot \mathrm{d} \mathbf{n}(s) / \mathrm{d} s .
$$

Let $\nu_{0}:=\inf _{0 \leq s \leq L} 1 /|c(s)|$. Then, for $\eta<\nu_{0}$,

$$
\forall \mathbf{x} \in \Omega_{m}^{\eta}, \exists !(s, \nu) \in\left[0, L[\times]-\eta / 2, \eta / 2\left[, \mathbf{x}=\mathbf{x}_{\Gamma}(s)+\nu \mathbf{n}(s) .\right.\right.
$$

Notice that $\mathbf{x}_{\Gamma}$ is the orthogonal projection of $\mathbf{x}$ on $\Gamma$. The couple $(s, \nu)$ will be referred to as curvilinear (or parametric) coordinates of $\mathrm{x} \in \Omega_{m}^{\eta}$ (with respect to $\Gamma$ ). Let $u$ be a function defined on $\Omega_{m}^{\eta}$ and let $\widetilde{u}:[0, L[\times]-\eta / 2, \eta / 2[$ be defined by

$$
\widetilde{u}(s, \nu):=u(\mathbf{x}),
$$

where $\mathbf{x}$ and $(s, \nu)$ satisfy equation (3.1). Then, we have

$$
\nabla u(\mathbf{x})=\frac{1}{1+\nu c} \partial_{s} \widetilde{u} \boldsymbol{\tau}+\partial_{\nu} \widetilde{u} \mathbf{n}=\frac{1}{1+\nu c} \nabla_{\tau} \widetilde{u}+\partial_{\nu} \widetilde{u} \mathbf{n}
$$


and for a tensor $\overline{\boldsymbol{A}}$ such that $\overline{\boldsymbol{A}} \boldsymbol{\tau}=A^{\tau} \boldsymbol{\tau}$ and $\overline{\boldsymbol{A}} \mathbf{n}=A^{n} \mathbf{n}$, we have

$$
\begin{aligned}
\operatorname{div}(\overline{\boldsymbol{A}} \nabla u(\mathbf{x})) & =\frac{1}{1+\nu c} \partial_{s}\left(\frac{1}{1+\nu c} A^{\tau} \partial_{s} \widetilde{u}\right)+\frac{1}{1+\nu c} \partial_{\nu}\left((1+\nu c) A^{n} \partial_{\nu} \widetilde{u}\right) \\
& =\frac{1}{1+\nu c} \operatorname{div}_{\tau}\left(\frac{1}{1+\nu c} A^{\tau} \nabla_{\tau} \widetilde{u}\right)+\frac{1}{1+\nu c} \partial_{\nu}\left((1+\nu c) A^{n} \partial_{\nu} \widetilde{u}\right) .
\end{aligned}
$$

\subsection{Scaling and formal asymptotic expansion}

In order to take into account the relatively small values of the diffusion tensor along the normal coordinate we choose the scaling

$$
\sigma_{m}^{n}=\kappa_{0} \eta
$$

while we assume that $\overline{\boldsymbol{\sigma}}_{e}, \overline{\boldsymbol{\sigma}}_{i}$ and $\sigma_{m}^{\tau}$ are independent from $\eta$. Physically, the condition (3.4) may be an appropriate choice for dMRI modeling in the case of thin myelin layers and high $b$-values (at high $b$-values, permeability effects/water exchange become more prominent). We also scale the membrane $\Omega_{m}^{\eta}$ with respect to $\eta$ and transform this domain into (the $\eta$ independent domain) $\Gamma \times]-1 / 2,1 / 2\left[\right.$ through the mapping $\mathbf{x} \mapsto\left(\mathbf{x}_{\Gamma}(s), \nu / \eta\right)$. Let us denote by $M_{\ell}$ the restriction of $M$ to the domain $\Omega_{\ell}$ for $\ell=e, i, m$. We then define $\widetilde{M}_{m}$ on $\left.\Gamma \times\right]-1 / 2,1 / 2[\times[0, \infty)$ as

$$
\widetilde{M}_{m}\left(\mathbf{x}_{\Gamma}, \xi, t\right):=M_{m}(\mathbf{x}, t)
$$

with $\xi:=\frac{\nu}{\eta}$ and $\mathbf{x}, \mathbf{x}_{\Gamma}$ and $\nu$ satisfy (3.1). Since the time plays only the role of a parameter in the process of establishing membrane transmission condition, we shall omit indicating this variable in the notation. We first observe

$$
\operatorname{div}\left(\overline{\boldsymbol{\sigma}}_{m} \nabla M_{m}\right)=\frac{1}{1+\eta \xi c} \operatorname{div}_{\tau}\left(\frac{1}{1+\eta \xi c} \sigma_{m}^{\tau} \nabla_{\tau} \widetilde{M}_{m}\right)+\frac{1}{\eta^{2}} \frac{1}{1+\eta \xi c} \partial_{\xi}\left((1+\eta \xi c) \eta \kappa_{0} \partial_{\xi} \widetilde{M}_{m}\right)
$$

and also notice that

$$
i \mathbf{q} \cdot \mathbf{x} f(t) M=i\left(\mathbf{q} \cdot \mathbf{x}_{\Gamma}+q^{n} \eta \xi\right) f(t) \widetilde{M} .
$$

Assuming that $\widetilde{M}_{m}$ has the asymptotic expansion

$$
\widetilde{M}_{m}\left(\mathbf{x}_{\Gamma}, \xi\right)=\sum_{k=0}^{\infty} \eta^{k} M_{m}^{k}\left(\mathbf{x}_{\Gamma}, \xi\right)
$$

for some functions $M_{m}^{k}$ defined on $\left.\Gamma \times\right]-1 / 2,1 / 2\left[\right.$, the Bloch-Torrey equation (multiplied by the factor $(1+\eta \xi)^{3}$ ) implies

$$
\begin{aligned}
\sum_{k=0}^{\infty} \eta^{k}\left[(1+\eta \xi)^{3} \partial_{t} M_{m}^{k}+(1+\eta \xi)^{3} i\left(\mathbf{q} \cdot \mathbf{x}_{\Gamma}\right.\right. & \left.+q^{n} \eta \xi\right) f(t) M_{m}^{k}-(1+\eta \xi) \operatorname{div}_{\tau}\left(\sigma_{m}^{\tau} \nabla_{\tau} M_{m}^{k}\right) \\
& \left.+\eta \xi \sigma_{m}^{\tau} \nabla_{\tau} M_{m}^{k} \nabla_{\tau} c-\frac{\kappa_{0}}{\eta}(1+\eta \xi)^{3} \partial_{\xi \xi}^{2} M_{m}^{k}-(1+\eta \xi)^{2} \kappa_{0} c \partial_{\xi} M_{m}^{k}\right]=0
\end{aligned}
$$

Then, by formal identification of powers of $\eta$, we obtain in particular for the first two terms

$$
\begin{aligned}
& \kappa_{0} \partial_{\xi \xi}^{2} M_{m}^{0}=0 \\
& \kappa_{0} \partial_{\xi \xi}^{2} M_{m}^{1}=\partial_{t} M_{m}^{0}+i \mathbf{q} \cdot \mathbf{x}_{\Gamma} f(t) M_{m}^{0}-\operatorname{div}_{\tau}\left(\sigma_{m}^{\tau} \nabla_{\tau} M_{m}^{0}\right)-\kappa_{0} c \partial_{\xi} M_{m}^{0}
\end{aligned}
$$




\subsection{Derivation of ADTC}

The expression of ADTC will be obtained from explicit expression of the solutions of (3.5) and (3.6) in terms of $\xi$ and using the continuity conditions (2.7) that can be written in terms of $\widetilde{M}_{m}$ as

$$
\begin{aligned}
\widetilde{M}_{m}\left(\mathbf{x}_{\Gamma},-\frac{1}{2}\right) & =M_{i}\left(\mathbf{x}_{\Gamma}-\frac{\eta}{2} \mathbf{n}\right), \widetilde{M}_{m}\left(\mathbf{x}_{\Gamma}, \frac{1}{2}\right)=M_{e}\left(\mathbf{x}_{\Gamma}+\frac{\eta}{2} \mathbf{n}\right) \\
\kappa_{0} \partial_{\xi} \widetilde{M}_{m}\left(\mathbf{x}_{\Gamma},-\frac{1}{2}\right) & =\overline{\boldsymbol{\sigma}}_{i} \nabla M_{i}\left(\mathbf{x}_{\Gamma}-\frac{\eta}{2} \mathbf{n}\right) \cdot \mathbf{n}, \kappa_{0} \partial_{\xi} \widetilde{M}_{m}\left(\mathbf{x}_{\Gamma}, \frac{1}{2}\right)=\overline{\boldsymbol{\sigma}}_{e} \nabla M_{e}\left(\mathbf{x}_{\Gamma}+\frac{\eta}{2} \mathbf{n}\right) \cdot \mathbf{n} .
\end{aligned}
$$

In order to relate these boundary conditions to the asymptotic expansion of $\widetilde{M}_{m}$ we postulate that for $\ell=i, e$

$$
M_{\ell}=\sum_{k=0}^{\infty} \eta^{k} M_{\ell}^{k}
$$

where the functions $M_{\ell}^{k}$ are defined on $\Omega_{\ell}$ and satisfy the Bloch-Torrey equation in $\Omega_{\ell}$. We shall distinguish two families of ATC according to the way we choose to match the three asymptotic expansions.

\subsubsection{A first family of ADTC}

A first family of ADTC is obtained by imposing the continuity conditions

$$
M_{m}^{k}\left(\mathbf{x}_{\Gamma},-\frac{1}{2}\right)=M_{i}^{k}\left(\mathbf{x}_{\Gamma}-\frac{\eta}{2} \mathbf{n}\right), M_{m}^{k}\left(\mathbf{x}_{\Gamma}, \frac{1}{2}\right)=M_{\mathrm{e}}^{k}\left(\mathbf{x}_{\Gamma}+\frac{\eta}{2} \mathbf{n}\right)
$$

and

$$
\kappa_{0} \partial_{\xi} M_{m}^{k}\left(\mathbf{x}_{\Gamma},-\frac{1}{2}\right)=\overline{\boldsymbol{\sigma}}_{i} \nabla M_{i}^{k}\left(\mathbf{x}_{\Gamma}-\frac{\eta}{2} \mathbf{n}\right) \cdot \mathbf{n}, \kappa_{0} \partial_{\xi} M_{m}^{k}\left(\mathbf{x}_{\Gamma}, \frac{1}{2}\right)=\overline{\boldsymbol{\sigma}}_{e} \nabla M_{\mathrm{e}}^{k}\left(\mathbf{x}_{\Gamma}+\frac{\eta}{2} \mathbf{n}\right) \cdot \mathbf{n}
$$

for all $k$, which is obtained from (3.7) by formal identification of powers of $\eta$. We remark that in this way the functions $M_{m}^{k}$ depends also on $\eta$. Let us introduce the notations

$$
\begin{aligned}
l\left\langle M^{k}\right\rangle_{\eta}\left(\mathbf{x}_{\Gamma}\right) & :=\frac{M_{\mathrm{e}}^{k}\left(\mathbf{x}_{\Gamma}+\frac{\eta}{2} \mathbf{n}\right)+M_{i}^{k}\left(\mathbf{x}_{\Gamma}-\frac{\eta}{2}\right)}{2} \\
{\left[M^{k}\right]_{\eta}\left(\mathbf{x}_{\Gamma}\right): } & =M_{\mathrm{e}}^{k}\left(\mathbf{x}_{\Gamma}+\frac{\eta}{2}\right)-M_{i}^{k}\left(\mathbf{x}_{\Gamma}-\frac{\eta}{2} \mathbf{n}\right)
\end{aligned}
$$

and similar definitions for $\left\langle\overline{\boldsymbol{\sigma}} \nabla M^{k} \cdot \mathbf{n}\right\rangle_{\eta}$ and $\left[\overline{\boldsymbol{\sigma}} \nabla M^{k} \cdot \mathbf{n}\right]_{\eta}$. We shall first express $M_{m}^{k}$ (for $k=0,1$ ) in terms of $\left\langle M^{k}\right\rangle_{\eta}$ and $\left[M^{k}\right]_{\eta}$ by solving with respect to $\xi$ equations (3.5) and (3.6) using the two boundary conditions in (3.8). We then obtain an interface condition by using the two boundary conditions in (3.9). We can already remark that the obtained interface condition will not be a standard interface condition on $\Gamma$ but will correspond to a condition that couples the boundary values at $\partial \Omega_{\mathrm{e}}^{\eta}$ and $\partial \Omega_{i}^{\eta}$.

First order term. From (3.5) and the boundary conditions in (3.8) one readily sees that

$$
M_{m}^{0}=\left\langle M^{0}\right\rangle_{\eta}+\xi\left[M^{0}\right]_{\eta} .
$$

We then immediately get from (3.9)

$$
\left[\overline{\boldsymbol{\sigma}} \nabla M^{0} \cdot \mathbf{n}\right]_{\eta}=0 \text { and }\left\langle\overline{\boldsymbol{\sigma}} \nabla M^{0} \cdot \mathbf{n}\right\rangle_{\eta}=\kappa_{0}\left[M^{0}\right]_{\eta} .
$$


Second order term. From (3.5) and (3.8) and using the expression (3.11) one gets

$$
M_{m}^{1}\left(\mathbf{x}_{\Gamma}, \xi\right)=D_{0}\left(\mathbf{x}_{\Gamma}\right)+D_{1}\left(\mathbf{x}_{\Gamma}\right) \xi+D_{2}\left(\mathbf{x}_{\Gamma}\right) \xi^{2}+D_{3}\left(\mathbf{x}_{\Gamma}\right) \xi^{3}
$$

where

$$
\begin{aligned}
D_{0}\left(\mathbf{x}_{\Gamma}\right) & :=\left\langle M^{1}\right\rangle_{\eta}-\frac{1}{8 \kappa_{0}}\left(\left(\partial_{t}+i \mathbf{q} \cdot \mathbf{x}_{\Gamma} f(t)\right)\left\langle M^{0}\right\rangle_{\eta}-\operatorname{div}_{\tau}\left(\sigma_{m}^{\tau} \nabla_{\tau}\left\langle M^{0}\right\rangle_{\eta}\right)-\kappa_{0} c\left[M^{0}\right]_{\eta}\right) \\
D_{1}\left(\mathbf{x}_{\Gamma}\right) & :=\left[M^{1}\right]_{\eta}-\frac{1}{24 \kappa_{0}}\left(\left(\partial_{t}+i \mathbf{q} \cdot \mathbf{x}_{\Gamma} f(t)\right)\left[M^{0}\right]_{\eta}-\operatorname{div}_{\tau}\left(\sigma_{m}^{\tau} \nabla_{\tau}\left[M^{0}\right]_{\eta}\right)\right) \\
D_{2}\left(\mathbf{x}_{\Gamma}\right) & :=\frac{1}{2 \kappa_{0}}\left(\left(\partial_{t}+i \mathbf{q} \cdot \mathbf{x}_{\Gamma} f(t)\right)\left\langle M^{0}\right\rangle_{\eta}-\operatorname{div}_{\tau}\left(\sigma_{m}^{\tau} \nabla_{\tau}\left\langle M^{0}\right\rangle_{\eta}\right)-\kappa_{0} c\left[M^{0}\right]_{\eta}\right) \\
D_{3}\left(\mathbf{x}_{\Gamma}\right) & :=\frac{1}{6 \kappa_{0}}\left(\left(\partial_{t}+i \mathbf{q} \cdot \mathbf{x}_{\Gamma} f(t)\right)\left[M^{0}\right]_{\eta}-\operatorname{div}_{\tau}\left(\sigma_{m}^{\tau} \nabla_{\tau}\left[M^{0}\right]_{\eta}\right)\right) .
\end{aligned}
$$

We then conclude from (3.9)

$$
\begin{aligned}
& {\left[\overline{\boldsymbol{\sigma}} \nabla M^{1} \cdot \mathbf{n}\right]_{\eta}=\left(\partial_{t}+i \mathbf{q} \cdot \mathbf{x}_{\Gamma} f(t)\right)\left\langle M^{0}\right\rangle_{\eta}-\operatorname{div}_{\tau}\left(\sigma_{m}^{\tau} \nabla_{\tau}\left\langle M^{0}\right\rangle_{\eta}\right)-c \kappa_{0}\left[M^{0}\right]_{\eta}} \\
& \left\langle\overline{\boldsymbol{\sigma}} \nabla M^{1} \cdot \mathbf{n}\right\rangle_{\eta}=\kappa_{0}\left[M^{1}\right]_{\eta}+\frac{1}{12}\left(\partial_{t}+i \mathbf{q} \cdot \mathbf{x}_{\Gamma} f(t)\right)\left[M^{0}\right]_{\eta}-\frac{1}{12} \operatorname{div}_{\tau}\left(\sigma_{m}^{\tau} \nabla_{\tau}\left[M^{0}\right]_{\eta}\right) .
\end{aligned}
$$

A first ADTC of order two. According to the conditions (3.12) and (3.14) and since $M_{\ell}=M_{\ell}^{0}+\eta M_{\ell}^{1}+\mathcal{O}\left(\eta^{2}\right)$ for $\ell=e, i$, we obtain the following interface approximate conditions

$$
\begin{aligned}
& {[\overline{\boldsymbol{\sigma}} \nabla M \cdot \mathbf{n}]_{\eta}=\eta\left(\left(\partial_{t}+i \mathbf{q} \cdot \mathbf{x}_{\Gamma} f(t)\right)\langle M\rangle_{\eta}-\operatorname{div}_{\tau}\left(\sigma_{m}^{\tau} \nabla_{\tau}\langle M\rangle_{\eta}\right)-c \kappa_{0}[M]_{\eta}\right)+\mathcal{O}\left(\eta^{2}\right)} \\
& \langle\overline{\boldsymbol{\sigma}} \nabla M \cdot \mathbf{n}\rangle_{\eta}=\kappa_{0}[M]_{\eta}+\frac{\eta}{12}\left(\left(\partial_{t}+i \mathbf{q} \cdot \mathbf{x}_{\Gamma} f(t)\right)[M]_{\eta}-\operatorname{div}_{\tau}\left(\sigma_{m}^{\tau} \nabla_{\tau}[M]_{\eta}\right)\right)+\mathcal{O}\left(\eta^{2}\right) .
\end{aligned}
$$

A membrane transmission condition of order 2 with respect to $\eta$ is then obtained from (3.15) by dropping the $\mathcal{O}\left(\eta^{2}\right)$ terms. However, it turns out that the obtained expression does not lead to a diffusion problem that respect an energy identity similar to the original problem. This energy identity is important as it is supposed to provide uniform stability with respect to $\eta$. This stability is the main ingredient that guarantee the convergence rate at the consistency order (see, e.g., $[10,15,16]$ for similar problems).

In order to obtain an expression of ADTC that respects an uniform stability with respect to $\eta$, we shall replace the term $\eta \kappa_{0}[M]_{\eta}$ by $\eta\langle\overline{\boldsymbol{\sigma}} \nabla M \cdot \mathbf{n}\rangle_{\eta}$ in the first equation of (3.15) and add $\frac{\eta}{4} c[\overline{\boldsymbol{\sigma}} \nabla M \cdot \mathbf{n}]_{\eta}$ to the left hand side of the second equation (3.15). These substitutions, that have been suggested by the following energy proof, indeed do not change the formal $O\left(\eta^{2}\right)$ order of the reminders. We therefore propose as second order ADTC the following conditions

$$
\left\{\begin{array}{l}
{[\overline{\boldsymbol{\sigma}} \nabla M \cdot \mathbf{n}]_{\eta}+\eta c\langle\overline{\boldsymbol{\sigma}} \nabla M \cdot \mathbf{n}\rangle_{\eta}=\eta\left(\left(\partial_{t}+i \mathbf{q} \cdot \mathbf{x}_{\Gamma} f(t)\right)\langle M\rangle_{\eta}-\operatorname{div}_{\tau}\left(\sigma_{m}^{\tau} \nabla_{\tau}\langle M\rangle_{\eta}\right)\right)} \\
\langle\overline{\boldsymbol{\sigma}} \nabla M \cdot \mathbf{n}\rangle_{\eta}+\frac{\eta}{4} c[\overline{\boldsymbol{\sigma}} \nabla M \cdot \mathbf{n}]_{\eta}=\kappa_{0}[M]_{\eta}+\frac{\eta}{12}\left(\left(\partial_{t}+i \mathbf{q} \cdot \mathbf{x}_{\Gamma} f(t)\right)[M]_{\eta}-\operatorname{div}_{\tau}\left(\sigma_{m}^{\tau} \nabla_{\tau}[M]_{\eta}\right)\right) .
\end{array}\right.
$$

Stability of ADTC (3.16). We shall outline the proof of an energy identity of the Bloch-Torrey equation

$$
\partial_{t} M(\mathbf{x}, t)+i \mathbf{q} \cdot \mathbf{x} f(t) M(\mathbf{x}, t)-\operatorname{div}(\sigma(\mathbf{x}) \nabla M(\mathbf{x}, t))=0 \quad \text { in } \Omega_{i}^{\eta} \cup \Omega_{\mathrm{e}}^{\eta} \times(0, T)
$$

with the second order membrane transmission condition (3.16) on $\Gamma$. We only consider (for notation simplicity) the case where the diffusion in the axons and the extra-cellular space is isotropic, that is $\overline{\boldsymbol{\sigma}}_{e}=\sigma_{e} \mathrm{I}$ and $\overline{\boldsymbol{\sigma}}_{i}=\sigma_{i} \mathrm{I}$ 
(where I denotes the identity). Using the variational formulation with the conjugate $\bar{M}$ of $M$ as test function, we obtain, denoting $\Omega^{\eta}:=\Omega_{i}^{\eta} \cup \Omega_{e}^{\eta}$,

$$
\frac{1}{2} \frac{\mathrm{d}}{\mathrm{d} t} \int_{\Omega^{\eta}}|M|^{2}+\int_{\Omega^{\eta}} \sigma|\nabla M|^{2}+\int_{\Gamma_{\mathrm{e}}^{\eta}} \mathfrak{R e}\left(\left(\sigma_{e} \nabla M^{e} \cdot \mathbf{n}\right) \overline{M^{e}}\right)-\int_{\Gamma_{i}^{\eta}} \mathfrak{R e}\left(\left(\sigma_{i} \nabla M^{i} \cdot \mathbf{n}\right) \overline{M^{i}}\right)=0,
$$

where $\mathfrak{R e}(z)$ denotes the real part of a complex number $z$. Using the changes of variables $\boldsymbol{y}=\mathbf{x}_{\Gamma}+\frac{\eta}{2} \mathbf{n}$ and $\boldsymbol{y}=\mathbf{x}_{\Gamma}-\frac{\eta}{2} \mathbf{n}$, we obtain the following equalities for a smooth enough function $f$ (and small enough $\eta$ )

$$
\int_{\partial \Omega_{e}^{\eta}} f \mathrm{~d} s(\boldsymbol{y})=\int_{\Gamma} \tilde{f}\left(1+\frac{\eta}{2} c\right) \mathrm{d} s(\mathbf{x}) \text { and } \int_{\partial \Omega_{i}^{\eta}} f \mathrm{~d} s(\boldsymbol{y})=\int_{\Gamma} \tilde{f}\left(1-\frac{\eta}{2} c\right) \mathrm{d} s(\mathbf{x}) .
$$

Hence, we obtain

$$
\frac{1}{2} \frac{\mathrm{d}}{\mathrm{d} t} \int_{\Omega^{\eta}}|M|^{2}+\int_{\Omega^{\eta}} \sigma|\nabla M|^{2}+\int_{\Gamma} \mathfrak{R e}\left(\left(1+\frac{\eta}{2} c\right)\left(\sigma_{e} \nabla M^{e} \cdot \mathbf{n}\right) \overline{M^{e}}\right)-\int_{\Gamma} \mathfrak{R e}\left(\left(1-\frac{\eta}{2} c\right)\left(\sigma_{i} \nabla M^{i} \cdot \mathbf{n}\right) \overline{M^{i}}\right)=0 .
$$

Using the fact that

we obtain

$$
a^{e} b^{e}-a^{i} b^{i}=\left(a^{e}-a^{i}\right)\left(\frac{b^{e}+b^{i}}{2}\right)+\left(b^{e}-b^{i}\right)\left(\frac{a^{e}+a^{i}}{2}\right)
$$

$$
\begin{aligned}
\frac{1}{2} \frac{\mathrm{d}}{\mathrm{d} t} \int_{\Omega^{\eta}}|M|^{2}+\int_{\Omega^{\eta}} \sigma|\nabla M|^{2}+\int_{\Gamma} \mathfrak{R e}(([\sigma \nabla M & \left.\left.\cdot \mathbf{n}]_{\eta}+\eta c\langle\sigma \nabla M \cdot \mathbf{n}\rangle_{\eta}\right)\langle\bar{M}\rangle_{\eta}\right) \\
& +\int_{\Gamma} \mathfrak{R e}\left(\left(\langle\sigma \nabla M \cdot \mathbf{n}\rangle_{\eta}+\frac{\eta}{4} c[\sigma \nabla M \cdot \mathbf{n}]_{\eta}\right)[\bar{M}]_{\eta}\right)=0 .
\end{aligned}
$$

Using the ADTC (3.16), we end up with

$$
\begin{aligned}
\frac{1}{2} \frac{\mathrm{d}}{\mathrm{d} t} \int_{\Omega^{\eta}}|M|^{2} & +\int_{\Omega^{\eta}} \sigma|\nabla M|^{2}+\kappa_{0} \int_{\Gamma}\left|[M]_{\eta}\right|^{2} \\
& +\eta\left(\frac{1}{2} \frac{\mathrm{d}}{\mathrm{d} t} \int_{\Gamma}\left|\langle M\rangle_{\eta}\right|^{2}+\int_{\Gamma} \sigma_{m}^{\tau}\left|\left\langle\nabla_{\tau} M\right\rangle_{\eta}\right|^{2}\right)+\frac{\eta}{12}\left(\frac{1}{2} \frac{\mathrm{d}}{\mathrm{d} t} \int_{\Gamma}\left|[M]_{\eta}\right|^{2}+\int_{\Gamma} \sigma_{m}^{\tau}\left|\left[\nabla_{\tau} M\right]_{\eta}\right|^{2}\right)=0 .
\end{aligned}
$$

Finally, using this energy estimate, we obtain the following proposition:

Proposition 3.1. If $\overline{\boldsymbol{\sigma}}_{e}=\sigma_{e} \mathrm{I}$ and $\overline{\boldsymbol{\sigma}}_{i}=\sigma_{i} \mathrm{I}$, then the energy estimate (3.20) holds. Moreover, if the initial data $M(\cdot, 0)=M_{\text {init }}$ belongs to $\mathrm{H}^{1}\left(\Omega_{\ell}^{\eta}\right)$, for $\ell=i$, e, then the Bloch-Torrey equation (3.17) with the $A D T C$ (3.16) admits a unique solution $M_{\ell} \in \mathrm{L}^{2}\left(0, T ; \mathrm{H}^{1}\left(\Omega_{\ell}^{\eta}\right)\right) \cap C^{0}\left(0, T ; \mathrm{L}^{2}\left(\Omega_{\ell}^{\eta}\right)\right)$ such that $[M]_{\eta}$ and $\langle M\rangle_{\eta}$ belong to $\mathrm{L}^{2}\left(0, T ; \mathrm{H}^{1}(\Gamma)\right) \cap C^{0}\left(0, T ; \mathrm{L}^{2}(\Gamma)\right)$.

Proof. Let us denote by

$$
\mathrm{H}\left(\Omega^{\eta}\right):=\left\{\varphi \in \mathrm{H}^{1}\left(\Omega_{i}^{\eta} \cup \Omega_{e}^{\eta}\right) ;[\varphi]_{\eta} \in \mathrm{H}^{1}(\Gamma) \text { and }\langle\varphi\rangle_{\eta} \in \mathrm{H}^{1}(\Gamma)\right\} .
$$

Proceeding as above, we obtain the following variational formulation of problem (3.17)-(3.16): for all $\varphi \in \mathrm{H}\left(\Omega^{\eta}\right)$ :

$$
\begin{aligned}
\int_{\Omega^{\eta}} \partial_{t} M \varphi+\int_{\Omega^{\eta}} i \mathbf{q} \cdot \mathbf{x} M \varphi+\int_{\Omega^{\eta}} & (\sigma \nabla M) \cdot \nabla \varphi+\kappa_{0} \int_{\Gamma}[M]_{\eta}[\varphi]_{\eta} \\
& +\eta \int_{\Gamma} \partial_{t}\langle M\rangle_{\eta}\langle\varphi\rangle_{\eta}+\eta \int_{\Gamma}\left(i \mathbf{q} \cdot \mathbf{x}_{\Gamma}\langle M\rangle_{\eta}\langle\varphi\rangle_{\eta}+\sigma_{m}^{\tau} \nabla_{\tau}\langle M\rangle_{\eta} \cdot \nabla_{\tau}\langle\varphi\rangle_{\eta}\right) \\
& \quad+\frac{\eta}{12} \int_{\Gamma} \partial_{t}[M]_{\eta}[\varphi]_{\eta}+\frac{\eta}{12} \int_{\Gamma}\left(i \mathbf{q} \cdot \mathbf{x}_{\Gamma}[M]_{\eta}[\varphi]_{\eta}+\sigma_{m}^{\tau} \nabla_{\tau}[M]_{\eta} \cdot \nabla_{\tau}[\varphi]_{\eta}\right)=0
\end{aligned}
$$


This problem can be seen as a heat type equation for the triple $\left(M,\langle M\rangle_{\eta},\langle\varphi\rangle_{\eta}\right)$ by identifying the variational space $\mathrm{H}\left(\Omega^{\eta}\right)$ with the space

$$
\left\{(\varphi, \psi, \chi) \in \mathrm{H}^{1}\left(\Omega_{i}^{\eta} \cup \Omega_{e}^{\eta}\right) \times \mathrm{H}^{1}(\Gamma) \times \mathrm{H}^{1}(\Gamma) ;[\varphi]_{\eta}=\psi \text { and }\langle\varphi\rangle_{\eta}=\chi\right\} .
$$

We then conclude using the classical variational theory for evolution equations of Lions (see for instance [26], Chap. 3, Thm. 4.1 and Rem. 4.3 or [25]).

A conservation property for the ADTC (3.16). In dMRI, the measured signal corresponds to $\int_{\Omega} M$ and the application of a diffusion-encoding magnetic field gradient $(\mathbf{q} \neq \mathbf{0})$ induces attenuation of this quantity compared to the case $\mathbf{q}=\mathbf{0}$ (no attenuation). It is therefore important to check that our approximate model does not induce artificial attenuation when $\mathbf{q}=\mathbf{0}$.

When $\mathbf{q}=\mathbf{0}$, we easily obtain that

$$
\int_{\Omega_{e}^{\eta}} \partial_{t} M_{e}+\int_{\Omega_{i}^{\eta}} \partial_{t} M_{i}+\int_{\partial \Omega_{e}^{\eta}} \overline{\boldsymbol{\sigma}} \nabla M_{e} \mathbf{n}-\int_{\partial \Omega_{i}^{\eta}} \overline{\boldsymbol{\sigma}} \nabla M_{i} \mathbf{n}=0
$$

Using the changes of variables $\boldsymbol{y}=\mathbf{x}_{\Gamma}+\frac{\eta}{2} \mathbf{n}$ and $\boldsymbol{y}=\mathbf{x}_{\Gamma}-\frac{\eta}{2} \mathbf{n}$ in the boundary integrals, we obtain

$$
\int_{\Omega_{e}^{\eta}} \partial_{t} M_{e}+\int_{\Omega_{i}^{\eta}} \partial_{t} M_{i}+\int_{\Gamma}[\overline{\boldsymbol{\sigma}} \nabla M \cdot \mathbf{n}]_{\eta}+\eta \int_{\Gamma} c\langle\overline{\boldsymbol{\sigma}} \nabla M \cdot \mathbf{n}\rangle_{\eta}=0 .
$$

Therefore, using ADTC (3.16),

$$
\int_{\Omega_{e}^{\eta}} \partial_{t} M_{e}+\int_{\Omega_{i}^{\eta}} \partial_{t} M_{i}+\int_{\Gamma} \eta \partial_{t}\langle M\rangle_{\eta}=0
$$

In conclusion, we have the following mass conservation property, for all $t>0$,

$$
\int_{\Omega_{e}^{\eta} \cup \Omega_{i}^{\eta}} M(\mathbf{x}, t)+\eta \int_{\Gamma}\langle M(\mathbf{x}, t)\rangle_{\eta}=\int_{\Omega_{e}^{\eta} \cup \Omega_{i}^{\eta}} M(\mathbf{x}, 0)+\eta \int_{\Gamma}\langle M(\mathbf{x}, 0)\rangle .
$$

\subsubsection{A second family of ADTC}

We have to notice that the previous $\operatorname{ADTC}(3.16)$ has to be imposed numerically on each interface $\partial \Omega_{m}^{\eta} \cap \partial \Omega_{e}^{\eta}$ and $\partial \Omega_{m}^{\eta} \cap \partial \Omega_{i}^{\eta}$. However, in this case, we have to numerically manage a difficulty: the vertices on these interfaces would have to be aligned. Given that making powerful finite element mesh generation tools is an active area of research, this is a difficulty that may be resolved by choosing a good mesh generator. For example the mesh generator "TetGen" [32] allows the specification of element vertices. To overcome this difficulty in another way, we present here some additional computations based on Taylor expansion of $M_{e}$ and $M_{i}$ in order to obtain new ADTC imposed on the middle $\Gamma$ of the membrane.

The second family of ADTC is obtained by using the previous postulate, for $\ell=i, e$,

$$
M_{\ell}=\sum_{k=0}^{\infty} \eta^{k} M_{\ell}^{k}
$$

and by using Taylor expansions of $M_{\ell}^{k}$ for all $k$, that is

$$
\begin{aligned}
& M_{\ell}^{k}\left(\mathbf{x}_{\Gamma}+\frac{\eta}{2} \mathbf{n}\right)=M_{\ell}^{k}\left(\mathbf{x}_{\Gamma}\right)+\frac{\eta}{2} \nabla M_{\ell}^{k}\left(\mathbf{x}_{\Gamma}\right) \cdot \mathbf{n}+\frac{\eta^{2}}{4} \nabla^{2} M_{\ell}^{k}\left(\mathbf{x}_{\Gamma}\right) \cdot \mathbf{n} \cdot \mathbf{n}+\mathcal{O}\left(\eta^{3}\right) \\
& M_{\ell}^{k}\left(\mathbf{x}_{\Gamma}-\frac{\eta}{2} \mathbf{n}\right)=M_{\ell}^{k}\left(\mathbf{x}_{\Gamma}\right)-\frac{\eta}{2} \nabla M_{\ell}^{k}\left(\mathbf{x}_{\Gamma}\right) \cdot \mathbf{n}+\frac{\eta^{2}}{4} \nabla^{2} M_{\ell}^{k}\left(\mathbf{x}_{\Gamma}\right) \cdot \mathbf{n} \cdot \mathbf{n}+\mathcal{O}\left(\eta^{3}\right) .
\end{aligned}
$$


Hence, using the continuity conditions (3.7) and a formal identification of powers of $\eta$, we obtain (for $k=0,1$ ):

$$
\begin{aligned}
M_{m}^{0}\left(\mathbf{x}_{\Gamma},-\frac{1}{2}\right) & =M_{i}^{0}\left(\mathbf{x}_{\Gamma}\right) \\
M_{m}^{0}\left(\mathbf{x}_{\Gamma}, \frac{1}{2}\right) & =M_{e}^{0}\left(\mathbf{x}_{\Gamma}\right) \\
M_{m}^{1}\left(\mathbf{x}_{\Gamma},-\frac{1}{2}\right) & =M_{i}^{1}\left(\mathbf{x}_{\Gamma}\right)-\frac{1}{2} \nabla M_{i}^{0}\left(\mathbf{x}_{\Gamma}\right) \cdot \mathbf{n} \\
M_{m}^{1}\left(\mathbf{x}_{\Gamma}, \frac{1}{2}\right) & =M_{e}^{1}\left(\mathbf{x}_{\Gamma}\right)+\frac{1}{2} \nabla M_{e}^{0}\left(\mathbf{x}_{\Gamma}\right) \cdot \mathbf{n}
\end{aligned}
$$

and

$$
\begin{aligned}
& \kappa_{0} \partial_{\xi} M_{m}^{0}\left(\mathbf{x}_{\Gamma}-\frac{1}{2}\right)=\overline{\boldsymbol{\sigma}}_{i} \nabla M_{i}^{0}\left(\mathbf{x}_{\Gamma}\right) \cdot \mathbf{n} \\
& \kappa_{0} \partial_{\xi} M_{m}^{0}\left(\mathbf{x}_{\Gamma}+\frac{1}{2}\right)=\overline{\boldsymbol{\sigma}}_{e} \nabla M_{e}^{0}\left(\mathbf{x}_{\Gamma}\right) \cdot \mathbf{n} \\
& \kappa_{0} \partial_{\xi} M_{m}^{1}\left(\mathbf{x}_{\Gamma}-\frac{1}{2}\right)=\overline{\boldsymbol{\sigma}}_{i} \nabla M_{i}^{1}\left(\mathbf{x}_{\Gamma}\right) \cdot \mathbf{n}-\frac{1}{2} \overline{\boldsymbol{\sigma}}_{i} \nabla^{2} M_{-}^{0}\left(\mathbf{x}_{\Gamma}\right) \cdot \mathbf{n} \cdot \mathbf{n} \\
& \kappa_{0} \partial_{\xi} M_{m}^{1}\left(\mathbf{x}_{\Gamma}+\frac{1}{2}\right)=\overline{\boldsymbol{\sigma}}_{e} \nabla M_{e}^{1}\left(\mathbf{x}_{\Gamma}\right) \cdot \mathbf{n}+\frac{1}{2} \overline{\boldsymbol{\sigma}}_{e} \nabla^{2} M_{+}^{0}\left(\mathbf{x}_{\Gamma}\right) \cdot \mathbf{n} \cdot \mathbf{n} .
\end{aligned}
$$

We recall the notations

$$
\begin{aligned}
\left\langle M^{k}\right\rangle\left(\mathbf{x}_{\Gamma}\right) & :=\frac{M_{\mathrm{e}}^{k}\left(\mathbf{x}_{\Gamma}\right)+M_{i}^{k}\left(\mathbf{x}_{\Gamma}\right)}{2} \\
{\left[M^{k}\right]\left(\mathbf{x}_{\Gamma}\right) } & :=M_{\mathrm{e}}^{k}\left(\mathbf{x}_{\Gamma}\right)-M_{i}^{k}\left(\mathbf{x}_{\Gamma}\right) .
\end{aligned}
$$

We then follow the same strategy than in the previous subsection: we first express $M_{m}^{k}$ (for $k=0,1$ ) in terms of $\left\langle M^{k}\right\rangle$ and $\left[M^{k}\right]$ by solving with respect to $\xi$ equations (3.5) and (3.6) using the previous boundary conditions in (3.22). We then obtain an interface condition by using the boundary conditions (3.23).

First order term. From (3.5) and the boundary conditions in (3.22) one finds exactly the same conditions as in Section 3.3.1:

$$
M_{m}^{0}=\left\langle M^{0}\right\rangle+\xi\left[M^{0}\right]
$$

and, from (3.23),

$$
\left[\overline{\boldsymbol{\sigma}} \nabla M^{0} \cdot \mathbf{n}\right]=0 \text { and }\left\langle\overline{\boldsymbol{\sigma}} \nabla M^{0} \cdot \mathbf{n}\right\rangle=\kappa_{0}\left[M^{0}\right]
$$

Second order term. Proceeding as in Section 3.3.1, one gets from (3.5) and (3.22) and using the expression (3.25),

$$
M_{m}^{1}\left(\mathbf{x}_{\Gamma}, \xi\right)=F_{0}\left(\mathbf{x}_{\Gamma}\right)+F_{1}\left(\mathbf{x}_{\Gamma}\right) \xi+F_{2}\left(\mathbf{x}_{\Gamma}\right) \xi^{2}+F_{3}\left(\mathbf{x}_{\Gamma}\right) \xi^{3}
$$


where

$$
\begin{aligned}
F_{0}\left(\mathbf{x}_{\Gamma}\right):= & \left\langle M^{1}\right\rangle+\frac{1}{4}\left[\nabla M^{0} \cdot \mathbf{n}\right]-\frac{1}{8 \kappa_{0}}\left(\left(\partial_{t}+i \mathbf{q} \cdot \mathbf{x}_{\Gamma} f(t)\right)\left\langle M^{0}\right\rangle\right. \\
& \left.-\operatorname{div}_{\tau}\left(\sigma_{m}^{\tau} \nabla_{\tau}\left\langle M^{0}\right\rangle\right)-\kappa_{0} c\left[M^{0}\right]\right) \\
F_{1}\left(\mathbf{x}_{\Gamma}\right):= & {\left[M^{1}\right]+\left\langle\nabla M^{0} \cdot \mathbf{n}\right\rangle-\frac{1}{24 \kappa_{0}}\left(\left(\partial_{t}+i \mathbf{q} \cdot \mathbf{x}_{\Gamma} f(t)\right)\left[M^{0}\right]-\operatorname{div}_{\tau}\left(\sigma_{m}^{\tau} \nabla_{\tau}\left[M^{0}\right]\right)\right) } \\
F_{2}\left(\mathbf{x}_{\Gamma}\right):= & \frac{1}{2 \kappa_{0}}\left(\left(\partial_{t}+i \mathbf{q} \cdot \mathbf{x}_{\Gamma} f(t)\right)\left\langle M^{0}\right\rangle-\operatorname{div}_{\tau}\left(\sigma_{m}^{\tau} \nabla_{\tau}\left\langle M^{0}\right\rangle\right)-\kappa_{0} c\left[M^{0}\right]\right) \\
F_{3}\left(\mathbf{x}_{\Gamma}\right):= & \frac{1}{6 \kappa_{0}}\left(\left(\partial_{t}+i \mathbf{q} \cdot \mathbf{x}_{\Gamma} f(t)\right)\left[M^{0}\right]-\operatorname{div}_{\tau}\left(\sigma_{m}^{\tau} \nabla_{\tau}\left[M^{0}\right]\right)\right) .
\end{aligned}
$$

One can notice that $F_{2}=D_{2}$ and $F_{3}=D_{3}$, where $D_{2}$ and $D_{3}$ are defined in (3.13).

We then conclude from (3.23)

$$
\begin{aligned}
{\left[\overline{\boldsymbol{\sigma}} \nabla M^{1} \cdot \mathbf{n}\right]+\left\langle\overline{\boldsymbol{\sigma}} \nabla^{2} M^{0} \cdot \mathbf{n} \cdot \mathbf{n}\right\rangle=} & \left(\partial_{t}+i \mathbf{q} \cdot \mathbf{x}_{\Gamma} f(t)\right)\left\langle M^{0}\right\rangle-\operatorname{div}_{\tau}\left(\sigma_{m}^{\tau} \nabla_{\tau}\left\langle M^{0}\right\rangle\right)-c \kappa_{0}\left[M^{0}\right] \\
\left\langle\overline{\boldsymbol{\sigma}} \nabla M^{1} \cdot \mathbf{n}\right\rangle+\frac{1}{4}\left[\overline{\boldsymbol{\sigma}} \nabla^{2} M^{0} \cdot \mathbf{n} \cdot \mathbf{n}\right]= & \kappa_{0}\left[M^{1}\right]+\kappa_{0}\left\langle\nabla M^{0} \cdot \mathbf{n}\right\rangle \\
& +\frac{1}{12}\left(\left(\partial_{t}+i \mathbf{q} \cdot \mathbf{x}_{\Gamma} f(t)\right)\left[M^{0}\right]-\operatorname{div}_{\tau}\left(\sigma_{m}^{\tau} \nabla_{\tau}\left[M^{0}\right]\right)\right) .
\end{aligned}
$$

Moreover, we have on $\Gamma$, for $\ell=i, e$,

$$
\overline{\boldsymbol{\sigma}}_{\ell} \nabla^{2} M_{\ell}^{0} \cdot \mathbf{n} \cdot \mathbf{n}=\partial_{t} M_{\ell}^{0}+i \mathbf{q} \cdot \mathbf{x}_{\Gamma} f(t) M_{\ell}^{0}-\operatorname{div}_{\tau}\left(\overline{\boldsymbol{\sigma}}_{\ell} \nabla_{\tau} M_{\ell}^{0}\right)-c \overline{\boldsymbol{\sigma}}_{\ell} \nabla M_{\ell}^{0} \cdot \mathbf{n} .
$$

Hence, using (3.26), we obtain

$$
\begin{aligned}
{\left[\overline{\boldsymbol{\sigma}} \nabla M^{1} \cdot \mathbf{n}\right]=} & \operatorname{div}_{\tau}\left(\left\langle\left(\overline{\boldsymbol{\sigma}}-\sigma_{m}^{\tau} \mathrm{I}\right) \nabla_{\tau} M^{0}\right\rangle\right) \\
\left\langle\overline{\boldsymbol{\sigma}} \nabla M^{1} \cdot \mathbf{n}\right\rangle= & \kappa_{0}\left[M^{1}\right]+\kappa_{0}\left\langle\nabla M^{0} \cdot \mathbf{n}\right\rangle-\frac{1}{6}\left(\left(\partial_{t}+i \mathbf{q} \cdot \mathbf{x}_{\Gamma} f(t)\right)\left[M^{0}\right]+\frac{1}{2} \operatorname{div}_{\tau}\left(\sigma_{m}^{\tau} \nabla_{\tau}\left[M^{0}\right]\right)\right) \\
& +\frac{1}{4} \operatorname{div}_{\tau}\left(\left[\overline{\boldsymbol{\sigma}} \nabla_{\tau} M^{0}\right]\right) .
\end{aligned}
$$

In order to obtain an energy identity (see below), we rewrite these conditions as

$$
\begin{aligned}
{\left[\overline{\boldsymbol{\sigma}} \nabla M^{1} \cdot \mathbf{n}\right]=} & \operatorname{div}_{\tau}\left(\left\langle\left(\overline{\boldsymbol{\sigma}}-\sigma_{m}^{\tau} \mathrm{I}\right) \nabla_{\tau} M^{0}\right\rangle\right) \\
\left\langle\overline{\boldsymbol{\sigma}} \nabla M^{1} \cdot \mathbf{n}\right\rangle= & \kappa_{0}\left[M^{1}\right]+\kappa_{0}\left\langle\nabla M^{0} \cdot \mathbf{n}\right\rangle-\frac{1}{6}\left(\left(\partial_{t}+i \mathbf{q} \cdot \mathbf{x}_{\Gamma} f(t)\right)\left[M^{0}\right]\right. \\
& \left.-\operatorname{div}_{\tau}\left(\sigma_{m}^{\tau} \nabla_{\tau}\left[M^{0}\right]\right)\right)+\frac{1}{4} \operatorname{div}_{\tau}\left(\left[\left(\overline{\boldsymbol{\sigma}}-\sigma_{m}^{\tau} \mathrm{I}\right) \nabla_{\tau} M^{0}\right]\right) .
\end{aligned}
$$

A second ADTC of order two. According to the conditions (3.26) and (3.29) and since $M_{\ell}=M_{\ell}^{0}+\eta M_{\ell}^{1}+$ $\mathcal{O}\left(\eta^{2}\right)$ for $\ell=e, i$, we obtain the following interface approximate conditions

$$
\begin{aligned}
{[\overline{\boldsymbol{\sigma}} \nabla M \cdot \mathbf{n}] } & =\eta \operatorname{div}_{\tau}\left(\left\langle\left(\overline{\boldsymbol{\sigma}}-\sigma_{m}^{\tau} \mathrm{I}\right) \nabla_{\tau} M\right\rangle\right)+\mathcal{O}\left(\eta^{2}\right) \\
\langle\overline{\boldsymbol{\sigma}} \nabla M \cdot \mathbf{n}\rangle & =\kappa_{0}[M]-\frac{\eta}{6} P([M])+\frac{\eta}{4} \operatorname{div}_{\tau}\left(\left[\left(\overline{\boldsymbol{\sigma}}-\sigma_{m}^{\tau} \mathrm{I}\right) \nabla_{\tau} M\right]\right)+\mathcal{O}\left(\eta^{2}\right),
\end{aligned}
$$

where

$$
P([M]):=\left(\partial_{t}+i \mathbf{q} \cdot \mathbf{x}_{\Gamma} f(t)\right)[M]-\operatorname{div}_{\tau}\left(\sigma_{m}^{\tau} \nabla_{\tau}[M]\right) .
$$


The ADTC resulting from (3.30) turns out to be unconditionally unstable (similarly to what observed in $[10,15]$ for a different problem). In order to obtain a stable problem we shall replace the operator $\kappa_{0}[M]-\frac{\eta}{6} P$ by a Padé approximation up to $O\left(\eta^{2}\right)$ terms (which is compatible with the ADTC order). More precisely, we introduce an auxiliary unknown $\Psi$ on $\Gamma$ that satisfies

$$
\left(1+\frac{\eta}{6 \kappa_{0}} P\right) \Psi=[M]
$$

in such a way that

$$
\langle\overline{\boldsymbol{\sigma}} \nabla M \cdot \mathbf{n}\rangle=\kappa_{0} \Psi+\frac{\eta}{4} \operatorname{div}_{\tau}\left(\left[\left(\overline{\boldsymbol{\sigma}}-\sigma_{m}^{\tau} \mathrm{I}\right) \nabla_{\tau} M\right]\right)-\frac{1}{2} \frac{\eta^{2}}{6^{2} \kappa_{0}^{2}} P(P(\Psi))+\mathcal{O}\left(\eta^{2}\right) .
$$

By neglecting all $\mathcal{O}\left(\eta^{2}\right)$ terms, we end up with a second order membrane transmission condition on $\Gamma$ in the following form:

$$
\begin{aligned}
{[\overline{\boldsymbol{\sigma}} \nabla M \cdot \mathbf{n}] } & =\eta \operatorname{div}_{\tau}\left(\left\langle\left(\overline{\boldsymbol{\sigma}}-\sigma_{m}^{\tau} \mathrm{I}\right) \nabla_{\tau} M\right\rangle\right), \\
\langle\overline{\boldsymbol{\sigma}} \nabla M \cdot \mathbf{n}\rangle & =\kappa_{0} \Psi+\frac{\eta}{4} \operatorname{div}_{\tau}\left(\left[\left(\overline{\boldsymbol{\sigma}}-\sigma_{m}^{\tau} \mathrm{I}\right) \nabla_{\tau} M\right]\right),
\end{aligned}
$$

where

$$
\left\{\begin{aligned}
\left(1+\frac{\eta}{6 \kappa_{0}} P\right) \Psi & =[M] \\
\Psi=0 & \text { at } t=0 .
\end{aligned}\right.
$$

Remark 3.2. We can note that, in the specific case where $\overline{\boldsymbol{\sigma}}_{e}=\overline{\boldsymbol{\sigma}}_{i}=\sigma_{m}^{\tau} \mathrm{I}$, the second order ADTC has the simple form

$$
\begin{aligned}
& {[\overline{\boldsymbol{\sigma}} \nabla M \cdot \mathbf{n}]=0} \\
& \langle\overline{\boldsymbol{\sigma}} \nabla M \cdot \mathbf{n}\rangle=\kappa_{0} \Psi .
\end{aligned}
$$

where $\Psi$ satisfies (3.32).

Stability of ADTC (3.31)-(3.32). Here again, for notation simplicity, we assume that $\overline{\boldsymbol{\sigma}}_{e}=\sigma_{e} \mathrm{I}$ and $\overline{\boldsymbol{\sigma}}_{i}=\sigma_{i} \mathrm{I}$ in this paragraph. Then, proceeding similarly to Section 3.3.1 (notice that here the volumetric equations are valid in $\Omega_{i} \cup \Omega_{e}$ ), we obtain

$$
\begin{aligned}
\frac{1}{2} \frac{\mathrm{d}}{\mathrm{d} t} \int_{\Omega}|M|^{2}+\int_{\Omega} \sigma|\nabla M|^{2}-\eta \int_{\Gamma} & \mathfrak{R e}\left(\left\langle\left(\sigma-\sigma_{m}^{\tau}\right) \nabla_{\tau} M\right\rangle \cdot\left\langle\nabla_{\tau} \bar{M}\right\rangle\right)+\kappa_{0} \int_{\Gamma}|\Psi|^{2} \\
& +\frac{\eta}{6} \frac{1}{2} \frac{\mathrm{d}}{\mathrm{d} t} \int_{\Gamma}|\Psi|^{2}+\frac{\eta}{6} \int_{\Gamma} \sigma_{m}^{\tau}\left|\nabla_{\tau} \Psi\right|^{2}-\frac{\eta}{4} \int_{\Gamma} \mathfrak{R e}\left(\left[\left(\sigma-\sigma_{m}^{\tau}\right) \nabla_{\tau} M\right] \cdot\left[\nabla_{\tau} \bar{M}\right]\right)=0 .
\end{aligned}
$$

Hence, using the fact that

$$
\begin{aligned}
& a^{e} b^{e}+a^{i} b^{i}=2\left(\frac{a^{e}+a^{i}}{2}\right)\left(\frac{b^{e}+b^{i}}{2}\right)+\frac{1}{2}\left(a^{e}-a^{i}\right)\left(b^{e}-b^{i}\right) \\
& a^{e} b^{e}-a^{i} b^{i}=\left(a^{e}-a^{i}\right)\left(\frac{b^{e}+b^{i}}{2}\right)+\left(\frac{a^{e}+a^{i}}{2}\right)\left(b^{e}-b^{i}\right),
\end{aligned}
$$

we have

$$
\begin{aligned}
\int_{\Gamma}\left\langle\left(\sigma-\sigma_{m}^{\tau}\right) \nabla_{\tau} M\right\rangle \cdot\left\langle\nabla_{\tau} \bar{M}\right\rangle & =\int_{\Gamma}\left\langle\sigma-\sigma_{m}^{\tau}\right\rangle\left|\left\langle\nabla_{\tau} M\right\rangle\right|^{2}+\frac{1}{4} \int_{\Gamma}\left[\sigma-\sigma_{m}^{\tau}\right]\left[\nabla_{\tau} M\right] \cdot\left\langle\nabla_{\tau} \bar{M}\right\rangle \\
\int_{\Gamma}\left[\left(\sigma-\sigma_{m}^{\tau}\right) \nabla_{\tau} M\right] \cdot\left[\nabla_{\tau} \bar{M}\right] & =\int_{\Gamma}\left[\sigma-\sigma_{m}^{\tau}\right]\left\langle\nabla_{\tau} M\right\rangle \cdot\left[\nabla_{\tau} \bar{M}\right]+\int_{\Gamma}\left\langle\sigma-\sigma_{m}^{\tau}\right\rangle\left|\left[\nabla_{\tau} M\right]\right|^{2}
\end{aligned}
$$


and then, we obtain

$$
\begin{aligned}
\frac{1}{2} \frac{\mathrm{d}}{\mathrm{d} t} \int_{\Omega}|M|^{2} & +\int_{\Omega} \sigma|\nabla M|^{2}+\kappa_{0} \int_{\Gamma}|\Psi|^{2}+\frac{\eta}{12} \frac{\mathrm{d}}{\mathrm{d} t} \int_{\Gamma}|\Psi|^{2}+\frac{\eta}{6} \int_{\Gamma} \sigma_{m}^{\tau}\left|\nabla_{\tau} \Psi\right|^{2} \\
& +\eta \int_{\Gamma}\left\langle\sigma_{m}^{\tau}-\sigma\right\rangle\left|\left\langle\nabla_{\tau} M\right\rangle\right|^{2}+\frac{\eta}{4} \int_{\Gamma}\left\langle\sigma_{m}^{\tau}-\sigma\right\rangle\left|\left[\nabla_{\tau} M\right]\right|^{2}-\frac{\eta}{2} \int_{\Gamma} \mathfrak{R e}\left([\sigma]\left[\nabla_{\tau} M\right] \cdot\left\langle\nabla_{\tau} \bar{M}\right\rangle\right)=0 .
\end{aligned}
$$

Therefore, the stability is guaranteed as soon as the positivity of the terms in the second line of the above identity is ensured. This holds if

$$
\left\langle\sigma_{m}^{\tau}-\sigma\right\rangle \geq 0 \quad \text { and } \quad 4\left\langle\sigma_{m}^{\tau}-\sigma\right\rangle^{2}-[\sigma]^{2} \geq 0 .
$$

Remark 3.3. We can notice that the stability is ensured if $\sigma_{e}=\sigma_{i}=\sigma_{m}^{\tau}$. We also notice that in the case $\sigma_{e}=\sigma_{i}=\sigma$ the stability requires $\sigma_{m}^{\tau} \geq \sigma$, which is compatible with the observations in [10] for the case of the wave equation.

Let us set

$$
\mathrm{H}(\Omega):=\left\{\varphi \in \mathrm{H}^{1}\left(\Omega_{i} \cup \Omega_{e}\right) ;[\varphi] \in \mathrm{H}^{1}(\Gamma) \text { and }\langle\varphi\rangle \in \mathrm{H}^{1}(\Gamma)\right\} .
$$

Then, we obtain the following proposition (proceeding in a similar manner as in the proof of Prop. 3.1):

Proposition 3.4. If $\overline{\boldsymbol{\sigma}}_{e}=\sigma_{e} \mathrm{I}$ and $\overline{\boldsymbol{\sigma}}_{i}=\sigma_{i} \mathrm{I}$, then the energy estimate (3.34) holds. Moreover, if the condi-

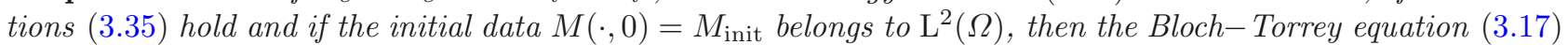
with the ADTC (3.31)-(3.32) admits a unique solution $(M, \Psi) \in \mathrm{L}^{2}(0, T ; \mathrm{H}(\Omega)) \times \mathrm{L}^{2}\left(0, T ; \mathrm{H}^{1}(\Gamma)\right)$ such that $(M, \Psi) \in C^{0}\left(0, T ; \mathrm{L}^{2}(\Omega)\right) \times C^{0}\left(0, T ; \mathrm{L}^{2}(\Gamma)\right)$.

A conservation property for the ADTC (3.31)-(3.32). Let us check again that our approximate model does not induce artificial attenuation in the case $\mathbf{q}=\mathbf{0}$. Indeed if $\mathbf{q}=\mathbf{0}$,

$$
\int_{\Omega_{e}} \partial_{t} M_{e}+\int_{\Omega_{i}} \partial_{t} M_{i}+\int_{\partial \Omega_{e}} \overline{\boldsymbol{\sigma}} \nabla M_{e} \cdot \mathbf{n}-\int_{\partial \Omega_{i}} \overline{\boldsymbol{\sigma}} \nabla M_{i} \cdot \mathbf{n}=0 .
$$

According to the new ADTC (3.31)-(3.32), $[\overline{\boldsymbol{\sigma}} \nabla M \cdot \mathbf{n}]=\eta \operatorname{div}_{\tau}\left(\left\langle\left(\overline{\boldsymbol{\sigma}}-\sigma_{m}^{\tau} \mathrm{I}\right) \nabla_{\tau} M\right\rangle\right)$. We therefore immediately obtain by integration by parts on $\Gamma$

$$
\int_{\Omega_{e}} \partial_{t} M_{e}+\int_{\Omega_{i}} \partial_{t} M_{i}=0 .
$$

In conclusion, for all $t>0$,

$$
\int_{\Omega_{e} \cup \Omega_{i}} M(\mathbf{x}, t)=\int_{\Omega_{e} \cup \Omega_{i}} M(\mathbf{x}, 0) .
$$

\section{NUMERICAL VALIDATION}

We numerically solve three models of the dMRI signal in the presence of thin layers:

(1) The original three-compartment model where $\Omega_{i}^{\eta}, \Omega_{m}^{\eta}, \Omega_{e}^{\eta}$ are linked by the interface conditions on $\Gamma_{i}^{\eta}$ and $\Gamma_{e}^{\eta}$ (see Eq. (2.7));

(2) The classical asymptotic two-compartment model where $\Omega^{e}$ and $\Omega^{i}$ are linked by the Isotropic Diffusion Transmission Condition (IDTC, see Eq. (2.10));

(3) The new asymptotic two-compartment model where $\Omega^{e}$ and $\Omega^{i}$ are linked by the Anisotropic Diffusion Transmission Condition (ADTC, see Eqs. (3.31)-(3.32)). 


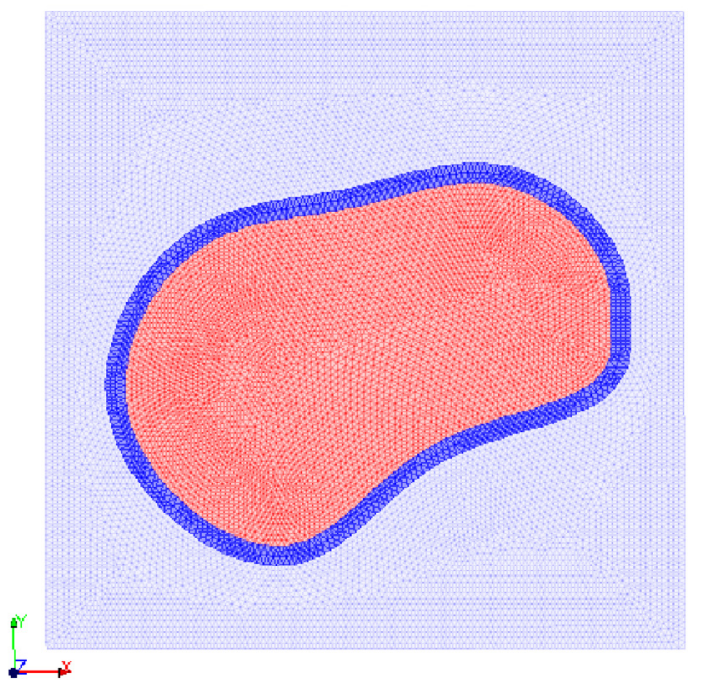

(a)

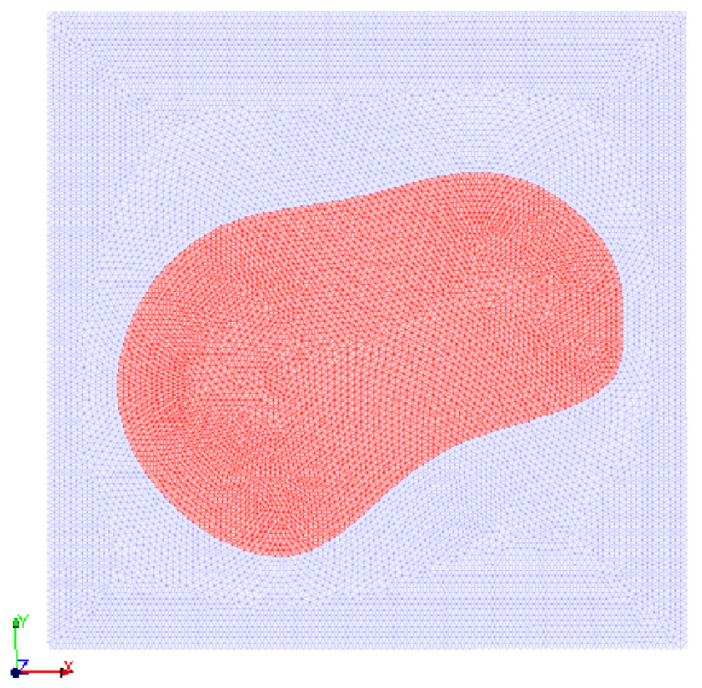

(b)

FiguRE 3. The three-compartment model (left) and the corresponding two-compartment model (right) for an irregular shaped axon.

We compute the dMRI signal associated with each of the three models. We compare the accuracy of the two asymptotic models in approximating the dMRI signal of the original three-compartment model as $\eta \rightarrow 0$. We note here that to implement the "ADTC", at each time step, we solve equation (3.32) on the interface (in $d-1$ dimension) with given jump $[M]$ to obtain $\Psi$. Then, we solve the Bloch-Torrey equation (2.1) with the Neumann boundary conditions (3.31) (in $d$ dimensions).

The simulations were performed in $d=2$ dimensions and the numerical method proposed in [28] was used and modified to solve the PDEs, where linear finite elements are coupled with the explicit Runge-Kutta Chebyshev (RKC) time stepping (see, e.g., $[33,36]$ ). The numerical code is implemented on FEniCS C++ platform and we used Salome 6.6.0 to generate finite element meshes. All simulations were performed on a Lenovo workstation (Intel(R) Xeon(R) CPU X3430@2.40GB), running the program as a serial code on Linux Ubuntu 10.04 LTS.

The computational domain $C$ is chosen to be $[-5 \mu \mathrm{m}, 5 \mu \mathrm{m}]^{2}$, containing an irregularly shaped axon. The thickness of the myelin sheath, $\eta$, is varied between $0.1 \mu \mathrm{m}$ and $1.5 \mu \mathrm{m}$. We set the maximum finite element diameter to $0.05 \mu \mathrm{m}$. The absolute tolerance $10^{-12}$ and relative tolerance $10^{-10}$ were set for the iterative linear solver GMRES, and the absolute and relative tolerance used for the RKC time stepping was $10^{-8}$. We define $\Gamma$ as the curve smoothly interpolated from 11 points in the xy-plane:

$$
\begin{gathered}
v_{1}=(4,0.1), \quad v_{2}=(4,0.2), \quad v_{3}=(4,0.3), \quad v_{4}=(4,0.4), \quad v_{5}=(2.3196,2.3883), \\
v_{6}=(-0.26237,2.0646), \quad v_{7}=(-2.4999,1.5231), \quad v_{8}=(-3.8547,-1.4071), \\
v_{9}=(-1.1845,-3.5318), \quad v_{10}=(1.2217,-1.8909), \quad v_{11}=(4,-0.33238) .
\end{gathered}
$$

Tangential and normal directions can be defined for each point of $\Gamma$. The myelin layer is defined by going from $\Gamma$ along the inward and outward normal directions a distance of $\eta / 2$ (see Fig. 3).

The diffusion in the axons and the extra-cellular space is supposed to be isotropic. The same intrinsic diffusion coefficient $\sigma_{i}=\sigma_{e}=3 \times 10^{-3} \mathrm{~mm}^{2} / \mathrm{s}$ is set for both compartments. Diffusion inside the layer is anisotropic with the tangential diffusion coefficient $\sigma_{m}^{\tau}=3 \times 10^{-3} \mathrm{~mm}^{2} / \mathrm{s}$ and transverse diffusion coefficient $\sigma_{m}^{n}$. We simulated two different values of $\kappa_{0}$ and varied the thickness $\eta$ between $0.1 \mu \mathrm{m}$ and $1.5 \mu \mathrm{m}$ for each value of $\kappa_{0}$. The transverse diffusion coefficient $\sigma_{m}^{n}$ is then computed from $\kappa_{0}$ and $\eta$ by equation (3.4). 


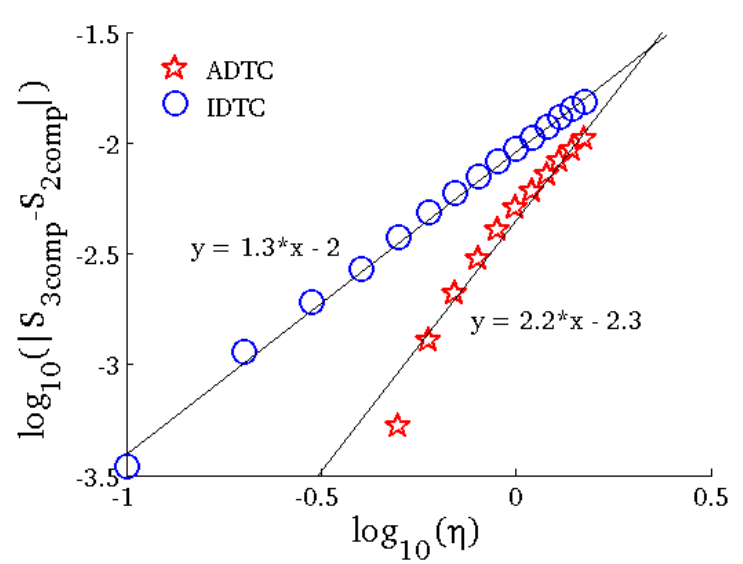

(a)

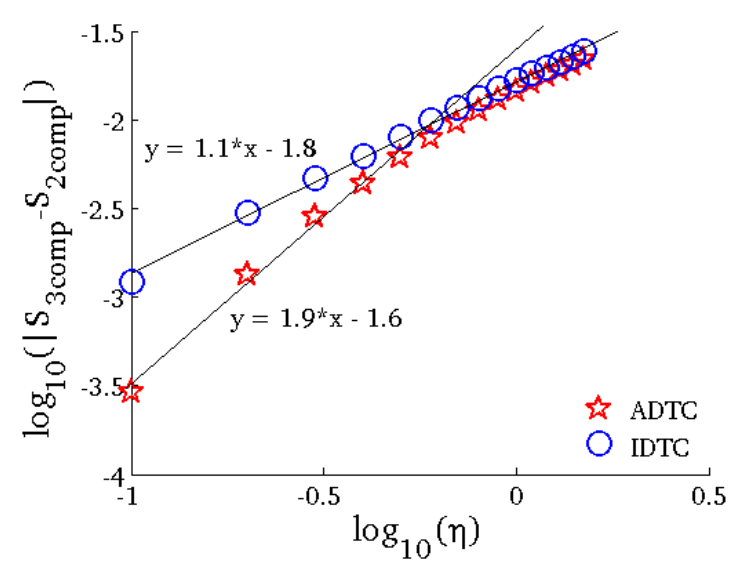

(c)

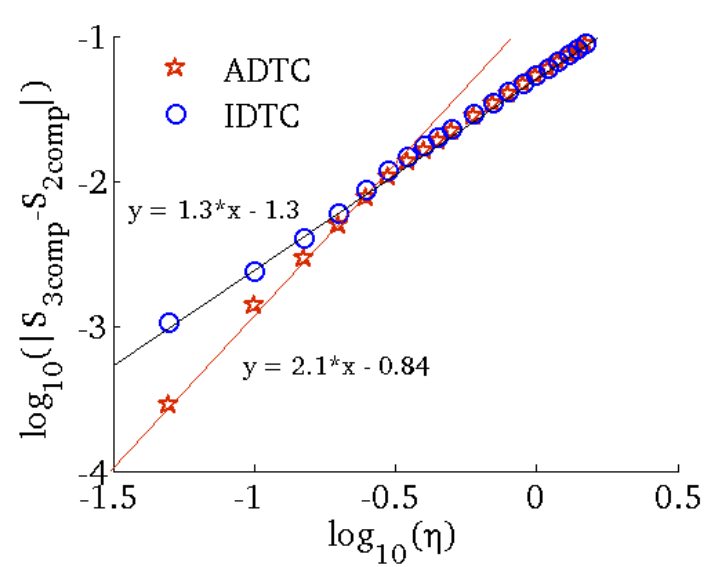

(b)

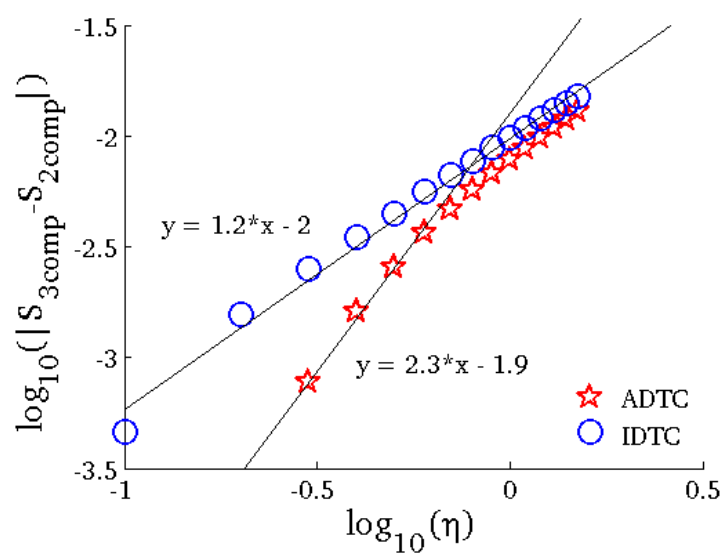

(d)

FIgURE 4. The signal of the new two compartment model with ADTC quadratically converges to that of the corresponding three-compartment model whereas the two compartment model with IDTC only gives the first order convergence. This result is shown at $\delta=\Delta=10 \mathrm{~ms}$ and $b=15000 \mathrm{~s} / \mathrm{mm}^{2}\left(\|\mathbf{q}\| \approx 4.74 \mathrm{~mm}^{-1} \mathrm{~s}^{-1}\right)$, for two different permeabilities $\kappa_{0}=10^{-5} \mathrm{~m} / \mathrm{s}$ (Fig. 4a and $\kappa_{0}=10^{-6} \mathrm{~m} / \mathrm{s}$ (Fig. 4b). For $\delta=\Delta=2 \mathrm{~ms}, \kappa_{0}=10^{-5} \mathrm{~m} / \mathrm{s}$, the quadratic convergence using ADTC and first order convergence using IDTC are shown for two $b$-values, $1000 \mathrm{~s} / \mathrm{mm}^{2}$ (Fig. 4c) and $2000 \mathrm{~s} / \mathrm{mm}^{2}$ (Fig. 4d).

The diffusion-encoding sequence is PGSE (see Eq. (2.2)), the gradient direction is $\frac{\mathbf{q}}{\|\mathbf{q}\|}=\frac{[1,1]}{\sqrt{2}}$. The uniform distribution of water protons is used for the initial condition:

$$
M(\mathbf{x}, 0)=1
$$

Reflecting boundary conditions are applied for the exterior boundaries of the computational domain $C$ :

$$
\overline{\boldsymbol{\sigma}} \nabla M \cdot \mathbf{n}=0 \quad \text { on } \partial C .
$$

First, we fix $\delta=\Delta=10 \mathrm{~ms}$ and $b=15000 \mathrm{~s} / \mathrm{mm}^{2}\left(\|\mathbf{q}\| \approx 4.74 \mathrm{~mm}^{-1} \mathrm{~s}^{-1}\right)$, and simulated $\kappa_{0}=10^{-5} \mathrm{~m} / \mathrm{s}$ (Fig. 4a) and $\kappa_{0}=10^{-6} \mathrm{~m} / \mathrm{s}$ (Fig. 4b). As expected, the signal of the two-compartment model with the ADTC 
converges quadratically to that of the corresponding three-compartment model whereas the two-compartment model with the IDTC only has first order convergence. Similarly, at $\delta=\Delta=2 \mathrm{~ms}, \kappa_{0}=10^{-5} \mathrm{~m} / \mathrm{s}$, we simulated two $b$-values, $1000 \mathrm{~s} / \mathrm{mm}^{2}$ (Fig. 4c) and $2000 \mathrm{~s} / \mathrm{mm}^{2}$ (Fig. 4d), and we can see that quadratic convergence is obtained using ADTC whereas the convergence is first order for IDTC. There appears to be a phenomenon of more rapid convergence than predicted by the theory as eta goes to 0 (as evidenced by a fit of 2.1 to 2.3 in the convergence order in Figs. $4 \mathrm{~b}-4 \mathrm{~d}$ ). The reasons of this phenomenon is probably due to the fact that our "exact" solution is not computed with sufficient accuracy since the used mesh is not sufficiently fine (resulting in a positive cancellation).

\section{Conclusions}

In this paper, we derived and validated a new transmission condition that accounts for anisotropic diffusion in thin layers. We showed theoretically that this condition is second order accurate in the layer thickness, whereas a more classical transmission condition is only first order accurate. The numerical simulations that we presented validate these results and show that our new model gives a better approximation of the dMRI signal than a simple transmission condition. We illustrated these transmission conditions in application to diffusion MRI where the diffusion inside the myelin sheath surrounding axons is smaller in the normal direction than the tangential direction.

The ADTC that we propose here simplifies the numerical solution of the dMRI model (i.e. solving the Bloch-Torrey equation at a microscopic scale) by removing the need to discretize the myelin sheath, which results in a computational saving that may be significant in three dimensions when simulating arbitrarily oriented white matter fibers.

\section{Appendix A. Justifichtion of the asymptotic model of Section 3.3.2}

We sketch here the proof of convergence for transmission problems established in previous sections which is somewhat technical but rather standard. We refer to $[10,15,16]$ for the investigation of more complex configurations. Our case is easier since one can rely on energy estimates to prove convergence results. The plan is the following. Firstly we establish error estimate between the exact solution of the problem and the truncated asymptotic expansion. Secondly we establish that, for the asymptotic model, an asymptotic expansion exists and provide error estimate with respect to truncated asymptotic expansions. Comparing the two asymptotic expansions, one observes that they match up to the order of consistency of the effective model. This provides, using a triangular inequality, the desired error estimate. We shall develop this only for the effective model of Section 3.3.2 which is the hardest case.

In the following, $0<T<\infty$ is a fixed time and $C$ denotes a generic constant that may have different values but is independent from the small parameter $\eta$ if the latter is taken sufficiently small. In the following, we assume that $M_{0}$ is a $C^{\infty}$ function on $\bar{\Omega}$ and that $\Gamma$ is a $C^{\infty}$ boundary. We also prescribe a homogeneous Dirichlet boundary condition on $\partial \Omega$ (other type of boundary conditions such as Neumann or Fourier homogeneous boundary conditions do not change the subsequent analysis).

\section{A.1. Error estimate between the exact solution and its truncated asymptotic expansion}

The formal identification of the asymptotic expansion terms allows the definition (by induction) of $M_{\ell}^{j}$, $\ell=i, e$, and $\widetilde{M}_{m}^{j}$ as smooth functions in time and space in respectively $\Omega_{\ell}$ and $]-1 / 2,1 / 2[\times \Gamma$. This technical step can be checked without any difficulty. Let us define the truncated series, for $\ell=i, e, m$,

$$
M_{\ell}^{k}(\mathbf{x}, t)=\sum_{j=0}^{k} \eta^{j} M_{\ell}^{j}(\mathbf{x}, t) \quad \mathbf{x} \in \Omega_{\ell}^{\eta}
$$


where $M_{m}^{j}(\mathbf{x}, t)=\widetilde{M}_{m}^{j}\left(\mathbf{x}_{\Gamma}, \nu / \eta, t\right)$ (see Sect. 3.2). Let us set $\mathrm{e}_{\ell}^{k}:=M-M_{\ell}^{k}$ in $\Omega_{\ell}^{\eta}$ for $\ell=i, e, m$. Indeed, from the formal expansion, one obtain, for $\ell=e, i$,

$$
\frac{\partial \mathrm{e}_{\ell}^{k}(\mathbf{x}, t)}{\partial t}+i \mathbf{q} \cdot \mathbf{x} f(t) \mathrm{e}_{\ell}^{k}(\mathbf{x}, t)-\operatorname{div}\left(\overline{\boldsymbol{\sigma}}(\mathbf{x}) \nabla \mathrm{e}_{\ell}^{k}(\mathbf{x}, t)\right)=0 \quad \text { in } \quad \Omega_{\ell}^{\eta},
$$

while

$$
\frac{\partial \mathrm{e}_{m}^{k}(\mathbf{x}, t)}{\partial t}+i \mathbf{q} \cdot \mathbf{x} f(t) \mathrm{e}_{m}^{k}(\mathbf{x}, t)-\operatorname{div}\left(\overline{\boldsymbol{\sigma}}(\mathbf{x}) \nabla \mathrm{e}_{m}^{k}(\mathbf{x}, t)\right)=\eta^{k} J_{\ell}^{\eta} \quad \text { in } \quad \Omega_{m}^{\eta},
$$

with zero initial data and boundary data on $\partial \Omega$, where $J_{\ell}^{\eta}$ as well as its time derivatives (that can be expressed in terms of $\widetilde{M}_{m}^{j}, j \leq k$ ) are bounded function in time and space uniformly with respect to $\eta$. Moreover, the jump conditions are such that, for $\ell=i, e$,

$$
\begin{aligned}
\mathrm{e}_{m}^{k}-\mathrm{e}_{\ell}^{k}=\eta^{k+1} \theta_{\ell}^{\eta} & \text { on } \quad \Gamma_{\ell}^{\eta} \\
\overline{\boldsymbol{\sigma}}_{m} \nabla \mathrm{e}_{m}^{k} \cdot \mathbf{n}-\overline{\boldsymbol{\sigma}}_{\ell} \nabla \mathrm{e}_{\ell}^{k} \cdot \mathbf{n}=\eta^{k+1} \beta_{\ell}^{\eta} & \text { on } \quad \Gamma_{\ell}^{\eta}
\end{aligned}
$$

where again $\theta_{\ell}^{\eta}$ and $\beta_{\ell}^{\eta}$ as well as their time derivatives are bounded in time and space uniformly with respect to $\eta$.

Then the main ingredients in deriving error estimates are the use of classical energy (stability) estimates for the heat equation, a Gronwall lemma type estimate and the following lemma.

Lemma A.1. There exists a constant $C$ independent from $\eta$ such that, for $\ell=i, e$,

$$
\|v\|_{\mathrm{H}^{1 / 2}\left(\Gamma_{\ell}^{\eta}\right)} \leq C \eta^{-1 / 2}\|v\|_{\mathrm{H}^{1}\left(\Omega_{m}^{\eta}\right)} \quad \forall v \in \mathrm{H}^{1}\left(\Omega_{m}^{\eta}\right)
$$

and

$$
\|\mathbf{v} \cdot \mathbf{n}\|_{\mathrm{H}^{-1 / 2}\left(\Gamma_{\ell}^{\eta}\right)} \leq C \eta^{-1 / 2}\left(\|\mathbf{v}\|_{\mathrm{L}^{2}\left(\Omega_{m}^{\eta}\right)}+\|\operatorname{div} \mathbf{v}\|_{\mathrm{L}^{2}\left(\Omega_{m}^{\eta}\right)}\right) \quad \forall \mathbf{v} \in \mathrm{H}\left(\operatorname{div}, \Omega_{m}^{\eta}\right) .
$$

Proof. Consider $\widetilde{v}$ defined on $]-1 / 2,1 / 2\left[\times \Gamma\right.$ from $v \in \mathrm{H}^{1}\left(\Omega_{m}^{\eta}\right)$ using the change of variable (see (3.1))

$$
\mathbf{x} \mapsto\left(\xi, \mathbf{x}_{\Gamma}\right) ; \quad \mathbf{x}=\mathbf{x}_{\Gamma}(s)+\eta \xi \mathbf{n}(s) .
$$

The first claim directly follows from the classical trace estimate

$$
\|\widetilde{v}( \pm 1 / 2, \cdot)\|_{\mathrm{H}^{1 / 2}(\Gamma)} \leq C\|\widetilde{v}\|_{\mathrm{H}^{1}(]-1 / 2,1 / 2[\times \Gamma)}
$$

and the change of variable.

Consider $\ell=i$ or $\ell=e$ and $\mathbf{v} \in \mathrm{H}\left(\operatorname{div}, \Omega_{m}^{\eta}\right)$. Let $\varphi \in \mathrm{H}^{1 / 2}\left(\Gamma_{\ell}^{\eta}\right)$ and extend $\varphi$ by 0 to the other part of the boundary of $\Omega_{m}^{\eta}$. Using the same technique as before, we also get the existence of a lifting $R(\varphi) \in \mathrm{H}^{1}\left(\Omega_{m}^{\eta}\right)$, such that

$$
\|R(\varphi)\|_{\mathrm{H}^{1}\left(\Omega_{m}^{\eta}\right)} \leq C \eta^{-1 / 2}\|\varphi\|_{\mathrm{H}^{1 / 2}\left(\Gamma_{\ell}^{\eta}\right)} .
$$

The second claim of the lemma then follows from the identity

$$
\langle\mathbf{v} \cdot \mathbf{n}, \varphi\rangle=\int_{\Omega_{m}^{\eta}}(\mathbf{v} \cdot \nabla R(\varphi)-R(\varphi) \operatorname{div} \mathbf{v})
$$

and the definition of $\mathrm{H}^{-1 / 2}\left(\Gamma_{\ell}^{\eta}\right)$ by duality.

Let us recall that by $\Omega:=\Omega_{\mathrm{e}}^{\eta} \cup \Omega_{i}^{\eta} \cup \Omega_{m}^{\eta}$. We are now in position to prove the first error estimate. 
Theorem A.2. For $k \geq 2$, there exists a constant $C$ independent from $\eta$ such that

$$
\left\|\mathrm{e}^{k}\right\|_{\mathrm{H}^{1}\left(0, T ; \mathrm{L}^{2}(\Omega)\right)}+\left(\int_{0}^{T} \int_{\Omega} \overline{\boldsymbol{\sigma}} \nabla \mathrm{e}^{k}(\mathbf{x}, t) \cdot \overline{\nabla \mathrm{e}^{k}(\mathbf{x}, t)}\right)^{1 / 2} \leq C \eta^{k}
$$

for sufficiently small $\eta$.

Proof. Let us denote by

$$
\mathcal{E}_{1}(t):=\frac{1}{2} \int_{\Omega}\left|\mathrm{e}^{k}(\mathbf{x}, t)\right|^{2} \mathrm{~d} \mathbf{x}+\int_{0}^{t} \int_{\Omega} \overline{\boldsymbol{\sigma}} \nabla \mathrm{e}^{k}(\mathbf{x}, \tau) \cdot \overline{\nabla \mathrm{e}^{k}(\mathbf{x}, \tau)} \mathrm{d} \mathbf{x} \mathrm{d} \tau
$$

and

$$
\mathcal{E}_{2}(t):=\frac{1}{2} \int_{\Omega} \overline{\boldsymbol{\sigma}} \nabla \mathrm{e}^{k}(\mathbf{x}, t) \cdot \overline{\nabla \mathrm{e}^{k}(\mathbf{x}, t)} \mathrm{d} \mathbf{x}+\int_{0}^{t} \int_{\Omega}\left|\frac{\partial \mathrm{e}^{k}(\mathbf{x}, \tau)}{\partial t}\right|^{2} \mathrm{~d} \mathbf{x} \mathrm{d} \tau .
$$

The first energy identity of the heat equation (obtained by multiplying the equations by $\overline{\mathrm{e}^{k}}$ then integrating by parts and integrating in time) applied to (A.1)-(A.4) implies

$$
\begin{aligned}
\mathcal{E}_{1}(t) \leq C \eta^{k}\left(\left(\int_{0}^{t} \mathcal{E}_{1}(\tau) \mathrm{d} \tau\right)^{1 / 2}+\eta\left(\int_{0}^{t}\left\|\left\langle\mathrm{e}^{k}\right\rangle(\tau)\right\|_{\mathrm{H}^{1 / 2}\left(\partial \Omega_{m}^{\eta}\right)}^{2} \mathrm{~d} \tau\right)^{1 / 2}\right. & \left.+\eta\left(\int_{0}^{t}\left\|\left\langle\overline{\boldsymbol{\sigma}} \nabla \mathrm{e}^{k} \cdot \mathbf{n}\right\rangle(\tau)\right\|_{\mathrm{H}^{-1 / 2}\left(\partial \Omega_{m}^{\eta}\right)}^{2} \mathrm{~d} \tau\right)^{1 / 2}\right) .
\end{aligned}
$$

The second energy identity of the heat equation (obtained by multiplying the equations by the time derivative of $\overline{\mathrm{e}^{k}}$ then integrating by parts in space and taking the integral in time, where integration by parts in time is performed for one of the boundary terms) applied to (A.1)-(A.4) implies

$$
\begin{aligned}
\mathcal{E}_{2}(t) \leq & C\left(\int_{0}^{t} \mathcal{E}_{1}(\tau) \mathrm{d} \tau\right)^{1 / 2} \sqrt{\mathcal{E}_{2}(t)}+C \eta^{k}\left(\sqrt{\mathcal{E}_{2}(t)}+\eta\left(\int_{0}^{t}\left\|\left\langle\mathrm{e}^{k}\right\rangle(\tau)\right\|_{\mathrm{H}^{1 / 2}\left(\partial \Omega_{m}^{\eta}\right)}^{2} \mathrm{~d} \tau\right)^{1 / 2}\right. \\
& \left.+\eta\left(\int_{0}^{t}\left\|\left\langle\overline{\boldsymbol{\sigma}} \nabla \mathrm{e}^{k} \cdot \mathbf{n}\right\rangle(\tau)\right\|_{\mathrm{H}^{-1 / 2}\left(\partial \Omega_{m}^{\eta}\right)}^{2} \mathrm{~d} \tau\right)^{1 / 2}+\eta\left\|\left\langle\mathrm{e}^{k}\right\rangle(t)\right\|_{\mathrm{H}^{1 / 2}\left(\partial \Omega_{m}^{\eta}\right)}\right) .
\end{aligned}
$$

From Lemma A.1, and since $\overline{\boldsymbol{\sigma}}$ is proportional to $\eta$ in $\Omega_{m}^{\eta}$, we get

$$
\left\|\left\langle\mathrm{e}^{k}\right\rangle(t)\right\|_{\mathrm{H}^{1 / 2}\left(\partial \Omega_{m}^{\eta}\right)}^{2} \leq C\left(\eta^{-2} \mathcal{E}_{2}(t)+\eta^{-1} \mathcal{E}_{1}(t)\right)
$$

and

$$
\int_{0}^{t}\left\|\left\langle\mathrm{e}^{k}\right\rangle(\tau)\right\|_{\mathrm{H}^{1 / 2}\left(\partial \Omega_{m}^{\eta}\right)}^{2} \mathrm{~d} \tau \leq C\left(\eta^{-2} \mathcal{E}_{1}(t)+\eta^{-1} \int_{0}^{t} \mathcal{E}_{1}(t)\right) .
$$

On the other hand, from (A.1) and (A.2), we obtain

$$
\int_{0}^{t}\left\|\operatorname{div}\left(\overline{\boldsymbol{\sigma}} \nabla \mathrm{e}^{k}\right)(\tau)\right\|_{\mathrm{L}^{2}(\Omega)}^{2} \mathrm{~d} \tau \leq C\left(\mathcal{E}_{2}(t)+\int_{0}^{t} \mathcal{E}_{1}(\tau) \mathrm{d} \tau+\eta^{2 k}\right) .
$$

From Lemma A.1 we then deduce

$$
\int_{0}^{t}\left\|\left\langle\overline{\boldsymbol{\sigma}} \nabla \mathrm{e}^{k} \cdot \mathbf{n}\right\rangle(\tau)\right\|_{\mathrm{H}^{-1 / 2}\left(\partial \Omega_{m}^{\eta}\right)}^{2} \mathrm{~d} \tau \leq C \eta^{-1}\left(\mathcal{E}_{2}(t)+\mathcal{E}_{1}(t)+\int_{0}^{t} \mathcal{E}_{1}(\tau) \mathrm{d} \tau+\eta^{2 k}\right) .
$$


After inserting (A.9), (A.10) and (A.11) into (A.7) and (A.8), one deduces that

$$
\mathcal{E}_{1}(t) \leq C \eta^{k}\left(\left(\int_{0}^{t} \mathcal{E}_{1}(\tau) \mathrm{d} \tau\right)^{1 / 2}+\sqrt{\mathcal{E}_{2}(t)}+\sqrt{\mathcal{E}_{1}(t)}+\eta^{k}\right)
$$

and

$$
\mathcal{E}_{2}(t) \leq C\left(\int_{0}^{t} \mathcal{E}_{1}(\tau) \mathrm{d} \tau\right)^{1 / 2} \sqrt{\mathcal{E}_{2}(t)}+C \eta^{k}\left(\left(\int_{0}^{t} \mathcal{E}_{1}(\tau) \mathrm{d} \tau\right)^{1 / 2}+\sqrt{\mathcal{E}_{2}(t)}+\sqrt{\mathcal{E}_{1}(t)}+\eta^{k}\right) .
$$

Setting

$$
\widetilde{\mathcal{E}}_{1}(t):=\int_{0}^{t} \mathcal{E}_{1}(\tau) \mathrm{d} \tau \text { and } \widetilde{\mathcal{E}}_{2}(t):=\int_{0}^{t} \mathcal{E}_{2}(\tau) \mathrm{d} \tau
$$

we respectively get, after integrating (A.12) and (A.13) between 0 and $t$ and using the fact that $\int_{0}^{t} \widetilde{\mathcal{E}}_{1}(\tau)^{1 / 2} \mathrm{~d} \tau \leq$ $t \widetilde{\mathcal{E}}_{1}(t)^{1 / 2}$,

$$
\widetilde{\mathcal{E}}_{1}(t) \leq C \eta^{k}\left(\sqrt{\widetilde{\mathcal{E}}_{2}(t)}+\sqrt{\widetilde{\mathcal{E}}_{1}(t)}+\eta^{k}\right)
$$

and

$$
\widetilde{\mathcal{E}}_{2}(t) \leq C \sqrt{\widetilde{\mathcal{E}}_{1}(t)} \sqrt{\widetilde{\mathcal{E}}_{2}(t)}+C \eta^{k}\left(\sqrt{\widetilde{\mathcal{E}}_{2}(t)}+\sqrt{\widetilde{\mathcal{E}}_{1}(t)}+\eta^{k}\right)
$$

We then deduce that

$$
\sqrt{\widetilde{\mathcal{E}}_{1}(t)}+\sqrt{\widetilde{\mathcal{E}}_{2}(t)} \leq C \eta^{k}
$$

Plugging this estimate in (A.12) and (A.13) to bound $\sqrt{\widetilde{\mathcal{E}}_{1}(t)}$ we then also deduce that

$$
\sqrt{\mathcal{E}_{1}(t)}+\sqrt{\mathcal{E}_{2}(t)} \leq C \eta^{k}
$$

which yields the desired estimate.

\section{A.2. Error estimate between the approximated solution and its truncated asymptotic expansion}

Let us now consider the solution of the asymptotic problem that we shall denote with a wide hat (in order to distinguish this solution from the solution of the original problem). The solutions $\widehat{M}$ and $\Psi$ satisfy

$$
\frac{\partial \widehat{M}(\mathbf{x}, t)}{\partial t}+i \mathbf{q} \cdot \mathbf{x} f(t) \widehat{M}(\mathbf{x}, t)-\operatorname{div}(\overline{\boldsymbol{\sigma}}(\mathbf{x}) \nabla \widehat{M}(\mathbf{x}, t))=0
$$

in $\Omega_{i} \cup \Omega_{e}$ together with the same initial conditions as the original solution and the jump conditions (3.31)-(3.32).

For simplicity we shall assume that $\overline{\boldsymbol{\sigma}}_{e}=\sigma_{e} \mathrm{I}$ and $\overline{\boldsymbol{\sigma}}_{i}=\sigma_{i}$ I. Then, using the stability estimate (3.34) of the approximate problem, we shall prove that this problem admits an asymptotic expansion of the form (for $\ell=i, e$ )

$$
\widehat{M}(\mathbf{x}, t)=\sum_{j=0}^{\infty} \eta^{j} \widehat{M}_{\ell}^{j}(\mathbf{x}, t), \quad \mathbf{x} \in \Omega_{\ell}
$$

and

$$
\Psi=\sum_{j=0}^{\infty} \eta^{j} \Psi_{\ell}^{j}(\mathbf{x}, t), \quad \mathbf{x} \in \Gamma
$$


Indeed the insertion of these ansatz into the equations of the asymptotic problem and formal identification of the same powers in $\eta$ allow the identification of the set of equations satisfied by $\widehat{M}_{\ell}^{j}$ and $\Psi_{\ell}^{j}$. These equations are inductive and allow the definition of these terms as regular functions independent from $\eta$. It is very easy to observe that $\widehat{M}_{\ell}^{j}$ verifies the same set of equations as $M_{\ell}^{j}$ for $\ell=i, e$ and $k=0,1$. We then conclude

$$
\widehat{M}_{\ell}^{0}=M_{\ell}^{0} \quad \text { and } \quad \widehat{M}_{\ell}^{1}=M_{\ell}^{1} .
$$

Consider now the truncated series, for $\ell=i, e$,

$$
\widehat{M}_{\ell}^{k}(\mathbf{x}, t)=\sum_{j=0}^{k} \eta^{j} \widehat{M}_{\ell}^{j}(\mathbf{x}, t), \quad \mathbf{x} \in \Omega_{\ell}^{\eta},
$$

and

$$
\Psi^{k}(\mathbf{x}, t)=\sum_{j=0}^{k} \eta^{j} \Psi^{j}(\mathbf{x}, t), \quad \mathbf{x} \in \Gamma .
$$

Let us set $\widehat{e}_{\ell}^{k}:=\widehat{M}_{\ell}-\widehat{M}_{\ell}^{k}$ in $\Omega_{\ell}^{\eta}$ for $\ell=i, e$ and $\psi^{k}:=\Psi-\Psi^{k}$ on $\Gamma$. From the formal expansion one can check that, for $\ell=e, i$ and $k \geq 2$,

$$
\frac{\partial \widehat{e}_{\ell}^{k}(\mathbf{x}, t)}{\partial t}+i \mathbf{q} \cdot \mathbf{x} f(t) \widehat{e}_{\ell}^{k}(\mathbf{x}, t)-\operatorname{div}\left(\overline{\boldsymbol{\sigma}}(\mathbf{x}) \nabla \widehat{e}_{\ell}^{k}(\mathbf{x}, t)\right)=0 \quad \text { in } \Omega_{\ell},
$$

with zero initial data and boundary data on $\partial \Omega$ and on $\Gamma$

$$
\begin{gathered}
\left\{\begin{aligned}
{\left[\overline{\boldsymbol{\sigma}} \nabla \widehat{e}^{k} \cdot \mathbf{n}\right] } & =\eta \operatorname{div}_{\tau}\left(\left\langle\left(\overline{\boldsymbol{\sigma}}-\sigma_{m}^{\tau} \mathrm{I}\right) \nabla_{\tau} \widehat{e}\right\rangle\right)+\eta^{k+1} \theta_{1}^{\eta}, \\
\left\langle\overline{\boldsymbol{\sigma}} \nabla \widehat{e}^{k} \cdot \mathbf{n}\right\rangle & =\kappa_{0} \psi^{k}+\frac{\eta}{4} \operatorname{div}_{\tau}\left(\left[\left(\overline{\boldsymbol{\sigma}}-\sigma_{m}^{\tau} \mathrm{I}\right) \nabla_{\tau} \widehat{e}^{k}\right]\right)+\eta^{k+1} \theta_{2}^{\eta},
\end{aligned}\right. \\
\left\{\begin{array}{r}
\left(1+\frac{\eta}{6 \kappa_{0}} P\right) \psi^{k}=\left[\widehat{e}^{k}\right]+\eta^{k+1} \theta_{3}^{\eta}, \\
\psi^{k}=0 \quad \text { at } t=0,
\end{array}\right.
\end{gathered}
$$

hold for some boundary terms $\theta_{i}^{\eta}$ that are regular and uniformly bounded with respect to $\eta$ as well as their time derivatives.

Theorem A.3. Assume that (3.35) is verified, then, for $k \geq 0$, there exists a constant $C$ independent from $\eta$ such that

$$
\left\|\widehat{e}^{k}\right\|_{\mathrm{L}^{2}\left(0, T ; \mathrm{H}^{1}(\Omega \backslash \Gamma)\right)} \leq C \eta^{k+1}
$$

for sufficiently small $\eta$.

Proof. Let us consider the energy

$$
\begin{aligned}
\mathcal{E}(t):=\frac{1}{2} \int_{\Omega}\left|\widehat{e}^{k}\right|^{2}+\int_{0}^{t} \int_{\Omega} \sigma\left|\nabla \widehat{e}^{k}\right|^{2}+\kappa_{0} \int_{0}^{t} \int_{\Gamma}\left|\psi^{k}\right|^{2}+\frac{\eta}{12} \int_{\Gamma}\left|\psi^{k}\right|^{2}+\frac{\eta}{6} \int_{0}^{t} \int_{\Gamma} \sigma_{m}^{\tau}\left|\nabla_{\tau} \psi^{k}\right|^{2} & \\
& +\eta \int_{0}^{t} \int_{\Gamma}\left\langle\sigma_{m}^{\tau}-\sigma\right\rangle\left|\left\langle\nabla_{\tau} \widehat{e}^{k}\right\rangle\right|^{2}+\frac{\eta}{4} \int_{0}^{t} \int_{\Gamma}\left\langle\sigma_{m}^{\tau}-\sigma\right\rangle\left|\left[\nabla_{\tau} \widehat{e}^{k}\right]\right|^{2} \\
& -\frac{\eta}{2} \int_{0}^{t} \int_{\Gamma} \mathfrak{R e}\left([\sigma]\left[\nabla_{\tau} \widehat{e}^{k}\right] \cdot\left\langle\nabla_{\tau} \overline{\bar{e}^{k}}\right\rangle\right) .
\end{aligned}
$$


Then, using the same calculations as those for establishing (3.34), we get from (A.17)-(A.19)

$$
\frac{\mathrm{d} \mathcal{E}}{\mathrm{d} t}(t)=\mathfrak{R e}\left(\int_{\Gamma} \eta^{k+1}\left(\theta_{1}^{\eta}\left[\overline{\widehat{e}^{k}}\right]+\theta_{2}^{\eta}\left\langle\overline{\widehat{e}^{k}}\right\rangle+\theta_{3}^{\eta} \overline{\psi^{k}}\right)\right),
$$

where $\mathfrak{R e}(z)$ denotes the real part of a complex number $z$. Moreover, since (3.35) holds, then

$$
\int_{0}^{t}\left\|\widehat{e}^{k}(\tau)\right\|_{\mathrm{H}^{1}(\Omega \backslash \Gamma)}^{2}+\left\|\psi^{k}(\tau)\right\|_{\mathrm{L}^{2}(\Gamma)}^{2} \mathrm{~d} \tau \leq C\left(\int_{0}^{t} \mathcal{E}(\tau) \mathrm{d} \tau+\mathcal{E}(t)\right) .
$$

We then deduce, from trace theorems that,

$$
\mathcal{E}(t) \leq C \eta^{k+1}\left(\left(\int_{0}^{t} \mathcal{E}(\tau) \mathrm{d} \tau\right)^{1 / 2}+\sqrt{\mathcal{E}(t)}\right) .
$$

We finally conclude, using similar arguments as at the end of the proof of Theorem A.2, that for sufficiently small $\eta$ that

$$
\sqrt{\mathcal{E}(t)} \leq C \eta^{k+1}
$$

which allows us to finish the proof of the theorem (using that (3.35) is verified).

\section{A.3. Error estimate between the exact solution and the approximated solution}

We are now in position to state the final convergence theorem.

Theorem A.4. Assume that (3.35) is verified, then for sufficiently small $\eta$,

$$
\|M-\widehat{M}\|_{\mathrm{L}^{2}\left(0, T ; \mathrm{H}^{1}\left(\Omega_{e} \cup \Omega_{i}\right)\right)} \leq C \eta^{2} .
$$

Proof. Using Theorem A.2 with $k=2$ we indeed deduce, using the triangular inequality, that

$$
\left\|M_{\ell}-M_{\ell}^{0}+\eta M_{\ell}^{1}\right\|_{\mathrm{L}^{2}\left(0, T ; \mathrm{H}^{1}\left(\Omega_{\ell}^{\eta}\right)\right)} \leq C \eta^{2}
$$

for $\ell=i, e$. We then easily conclude using Theorem A.3 with $k=1$ and (A.16).

\section{REFERENCES}

[1] Y. Achdou, O. Pironneau and F. Valentin, Effective boundary conditions for laminar flows over periodic rough boundaries. J. Comput. Phys. 147 (1998) 187-218.

[2] B. Aslanyürek, H. Haddar and H. Şahintürk, Generalized impedance boundary conditions for thin dielectric coatings with variable thickness. Wave Motion 48 (2011) 681-700.

[3] Y. Assaf, R.Z. Freidlin, G.K. Rohde and P.J. Basser, New modeling and experimental framework to characterize hindered and restricted water diffusion in brain white matter. Magn. Reson. Med. 52 (2004) 965-978.

[4] W.Y. Aung, S. Mar and T.L. Benzinger, Diffusion tensor MRI as a biomarker in axonal and myelin damage. Imaging Med. 5 (2013) 427-440.

[5] C. Beaulieu, The basis of anisotropic water diffusion in the nervous system - a technical review. NMR Biomed. 15 (2002) $435-455$.

[6] A. Bendali and K. Lemrabet, The effect of a thin coating on the scattering of a time-harmonic wave for the Helmholtz equation. SIAM J. Appl. Math. 56 (1996) 1664-1693.

[7] I.E. Biton, I.D. Duncan and Y. Cohen, High b-value q-space diffusion MRI in myelin-deficient rat spinal cords. Magn. Reson. Imaging 24 (2006) 161-166.

[8] S. Chun, H. Haddar and J.S. Hesthaven, High-order accurate thin layer approximations for time-domain electromagnetics, Part II: transmission layers. J. Comput. Appl. Math. 234 (2010) 2587-2608.

[9] J. Coatléven, H. Haddar and J. Li, A macroscopic model including membrane exchange for diffusion MRI. SIAM J. Appl. Math. 74 (2014) 516-546.

[10] B. Delourme, H. Haddar and P. Joly, Approximate models for wave propagation across thin periodic interfaces. J. Math. Pures Appl. 98 (2012) 28-71. 
[11] M. Duruflé, V. Péron and C. Poignard, Thin Layer Models for Electromagnetism. Commun. Comput. Phys. 16 (2014) $213-238$.

[12] J. Farrell, Q-space Diffusion Imaging of Axon and Myelin Damage in the Human and Rat Spinal Cord. Johns Hopkins University (2009).

[13] R. Fox, T. Cronin, J. Lin, X. Wang, K. Sakaie, D. Ontaneda, S. Mahmoud, M. Lowe and M. Phillips, Measuring myelin repair and axonal loss with diffusion tensor imaging. Am. J. Neuroradiology 32 (2011) 85-91.

[14] H. Haddar and P. Joly, Effective boundary conditions for thin ferromagnetic coatings. Asymptotic analysis of the 1D model. Asymptot. Anal. 2 (2001) 127-160.

[15] H. Haddar and P. Joly, Stability of thin layer approximation of electromagnetic waves scattering by linear and nonlinear coatings. J. Comput. Appl. Math. 143 (2002) 201-236.

[16] H. Haddar, P. Joly and H.-M. Nguyen, Generalized impedance boundary conditions for scattering by strongly absorbing obstacles: the scalar case. Math. Models Methods Appl. Sci. 15 (2005) 1273-1300.

[17] H. Haddar, P. Joly and H.-M. Nguyen, Generalized impedance boundary conditions for scattering problems from strongly absorbing obstacles: The case of Maxwell's equations. Math. Models Methods Appl. Sci. 18 (2008) 1787-1827.

[18] M.A. Horsfield and D.K. Jones, Applications of diffusion-weighted and diffusion tensor MRI to white matter diseases, a review. NMR Biomed. 15 (2002) 570-577.

[19] W. Jäger and A. Mikelić, On the roughness-induced effective boundary conditions for an incompressible viscous flow. J. Differ. Equ. 170 (2001) 96-122.

[20] H. Johansen-Berg and T. Behrens, Diffusion MRI: From quantitative measurement to in vivo neuroanatomy. Elsevier Science (2013).

[21] D. Jones, Diffusion MRI: theory, methods, and applications. Oxford University Press, USA (2010).

[22] L.J. Lanyon, Neuroimaging - Methods, in: Diffusion tensor imaging: structural connectivity insights, limitations and future directions. Edited by Peter Bright. InTech (2012).

[23] D. Le Bihan and H. Johansen-Berg, Diffusion MRI at 25: Exploring brain tissue structure and function. NeuroImage 61 (2012) $324-34$.

[24] D. LeBihan, The wet mind: water and functional neuroimaging. Phys. Med. Biol. 52 (2007).

[25] J.-L. Lions, Quelques remarques sur les équations différentielles opérationnelles du $1^{\text {er }}$ ordre. Rend. Sem. Mat. Univ. Padova 33 (1963) 213-225.

[26] J.-L. Lions and E. Magenes, Non-homogeneous boundary value problems and applications, Vol. I. Translated from the French by P. Kenneth. Die Grundl. Math. Wiss., Band 181. Springer-Verlag, New York-Heidelberg (1972).

[27] S.E. Maier, Y. Sun and R.V. Mulkern, Diffusion imaging of brain tumors. NMR Biomed. 23 (2010) 849-864.

[28] D.V. Nguyen, J.-R. Li, D. Grebenkov and D.L. Bihan, A finite elements method to solve the Bloch-Torrey equation applied to diffusion magnetic resonance imaging. J. Comput. Phys. 263 (2014) 283-302.

[29] R. Perrussel and C. Poignard, Asymptotic expansion of steady- state potential in a high contrast medium with a thin resistive layer. Appl. Math. Comput. 221 (2013) 48-65.

[30] C. Poignard, Generalized impedance boundary condition at high frequency for a domain with thin layer: the circular case. Appl. Anal. 86 (2007) 1549-1568.

[31] R. Quarles, W. Macklin and P. Morell, Basic neurochemistry: molecular, cellular and medical aspects. In: Myelin formation, structure and biochemistry. Elsevier Science (2006).

[32] H. Si, TetGen, a Delaunay-based quality tetrahedral mesh generator. ACM Trans. Math. Softw. Art. 4 (2015) 11, 36.

[33] B.P. Sommeijer, L.F. Shampin and J.G. Verwer, RKC: an explicit solver for parabolic PDEs. J. Comput. Appl. Math. 88 (1998) 315-326.

[34] E.O. Stejskal and J.E. Tanner, Spin diffusion measurements: Spin echoes in the presence of a time-dependent field gradient. J. Chem. Phys. 42 (1965) 288-292.

[35] H. Torrey, Bloch equations with diffusion terms. Phys. Rev. Online Archive (Prola) 104 (1956) 563-565.

[36] J.G. Verwer, W.H. Hundsdorfer and B.P. Sommeijer, Convergence properties of the Runge-Kutta-Chebyshev method. Numer. Math. 57 (1990) 157-178. 